\title{
Die historische Rechtsvergleichung und das europäische Versicherungsrecht
}

\author{
Von
}

\section{Phillip Hellwege*)}

\begin{abstract}
Zusammenfassung: Der Beitrag zeigt die Grundlagen und Perspektiven einer historisch-vergleichenden Bearbeitung des Versicherungsrechts auf. Dafür werden die nationalen Forschungsstände zur Versicherungsrechtsgeschichte sowie die möglichen Verknüpfungen zwischen den nationalen Entwicklungen herausgearbeitet. Drei Beispiele, die darlegen, dass eine historisch-vergleichende Bearbeitung des Versicherungsrechts möglich und lohnend ist, runden den Beitrag ab.
\end{abstract}

I. Einleitung. - II. Nationale Forschungsstände. - III. Mögliche Verknüpfungen der nationalen Entwicklungen; 1. Versicherungspraxis; 2. Literatur; 3. Gesetzgebung; 4. Rechtsprechung; 5. Allgemeines Vertragsrecht. - IV. Drei Beispiele einer historisch-vergleichenden Bearbeitung des Versicherungsrechts; 1. Verhaltensanforderungen im Lebensversicherungswesen; a) Entwicklung in England; b) Entwicklung in Deutschland; c) Zwischenergebnis; 2. Verhaltensanforderungen im Feuerversicherungswesen; a) Entwicklung in England; b) Entwicklung in Deutschland; c) Zwischenergebnis; 3. Rechtsprechung zu Verhaltensanforderungen im Feuerversicherungswesen; a) Entwicklung in England; b) Entwicklung in Deutschland; c) Zwischenergebnis. - V. Zusammenfassung.

\section{Einleitung}

Als sich in den 1990er-Jahren die Aufgabe stellte, ein europäisches Privatrecht zu schaffen, bildeten sich rasch verschiedene Ansätze heraus ${ }^{1}$ ): Die einen propagierten eine vergleichende Herangehensweise und empfahlen, die mitgliedstaatlichen Rechte in den Blick zu nehmen, um Regeln zu erarbeiten, die allen Rechten Europas gemein sind²). Andere plädierten dafür, das

*) Der Beitrag basiert auf der Antrittsvorlesung des Autors vom 3. 7. 2012 in Augsburg.

1) Zum Folgenden Nils Jansen, Europäisches Privatrecht, in: Jürgen Basedow et al. (Hgg.), Handwörterbuch des Europäischen Privatrechts, 2009, 548-551.

$\left.{ }^{2}\right)$ Ole Lando, Principles of European Contract Law, American Journal of Comparative Law 40 (1992), 573-585, 579. 
Unionsprivatrecht als Ausgangspunkt zu wählen, um aus ihm Prinzipien eines europäischen Privatrechts herauszudestillieren ${ }^{3}$ ). Wieder andere traten dafür ein, den zuerst genannten Ansatz um eine historische Dimension zu erweitern ${ }^{4}$ ): Die gemeinsamen Wurzeln der europäischen Rechte müssten freigelegt und es müsste nachverfolgt werden, wie und warum sich die einzelnen Rechte seit ihrer Nationalisierung auseinanderentwickelt haben. Was zunächst nach einer Konkurrenz verschiedener Forschungsansätze aussah, ist inzwischen zu einem befruchtenden Miteinander geworden.

Keine Einigkeit besteht weiterhin über das Verhältnis von europäischer Privatrechtswissenschaft und europäischem Privatrecht ${ }^{5}$ ). Viele betonen, dass es zunächst nur um die Schaffung einer gemeinsamen Wissenschaft gehe $\left.{ }^{6}\right)$. Die bereits entstandenen Regelwerke, wie die Principles of European Contract $\mathrm{Law}^{7}$ ), seien bloße Textgrundlagen, um die sich eine solche Wissenschaft bilden könne. Derzeit befinde sich eine europäische Privatrechtswissenschaft noch in den Anfängen. Und trotz einheitlicher Textgrundlagen bleibe sie zunächst eine im Kern komparative Wissenschaft. Ein kodifizierbares europäisches Privatrecht müsse sich organisch aus der europäischen Privatrechtswissenschaft heraus entwickeln.

Andere streben eine rasche Kodifizierung an und verstehen die erwähnten Regelwerke als akademische Vorentwürfe eines solchen legislativen Aktes $^{8}$ ). Das europäische Privatrecht soll sich demnach nicht organisch aus einer (historisch-)vergleichenden europäischen Privatrechtswissenschaft entwickeln, sondern beide entstünden fast zeitgleich. Die europäische Privatrechtswissenschaft wird sich als Folge, so könnte man meinen, früh auf die bloße dogmatische Durchdringung einer eigenen Rechtsmasse konzentrieren können, ohne (historisch-)vergleichend arbeiten zu müssen.

Nimmt man die verschiedenen Regelungsmaterien in den Blick, so fällt auf, dass die beschriebene Methodenvielfalt und der aufgezeigte Konflikt

${ }^{3}$ ) Hans-W. Micklitz, Perspektiven eines Europäischen Privatrechts, Zeitschrift für Europäisches Privatrecht [ZEuP] 6 (1998), 253-276.

${ }^{4}$ ) Reinhard Zimmermann, Das römisch-kanonische ius commune als Grundlage europäischer Rechtseinheit, Juristenzeitung 1992, 8-20.

5) Zusammenfassend Martin Schmidt-Kessel, Europäisches Zivilgesetzbuch, in: Handwörterbuch (Fn. 1), 551-555.

$\left.{ }^{6}\right)$ So Reinhard Zimmermann, Savignys Vermächtnis, 1998, 54ff.

$\left.{ }^{7}\right)$ Ole Lando/Hugh Beale (Hgg.), Principles of European Contract Law, Parts I and II, 2000; Ole Lando et al. (Hgg.), Principles of European Contract Law, Part III, 2003.

$\left.{ }^{8}\right)$ So Martijn W. Hesselink, The Common Frame of Reference as a Source of European Private Law, Tulane Law Review 83 (2009), 919-971, 923. 
nur in den Kernmaterien des Bürgerlichen Rechts existieren. Im Übrigen dominieren einzelne Ansätze. So stellt sich die Aufgabe der Schaffung einer europäischen Rechtswissenschaft bzw. eines europäischen Rechts auch im Versicherungsrecht ${ }^{9}$ ). Doch fehlt hier bisher eine historische Perspektive. Man arbeitet unter Einbeziehung des Acquis communautaire vor allem vergleichend ${ }^{10}$ ), hat so die Principles of European Insurance Contract Law (PEICL) ${ }^{11}$ ) geschaffen und versteht diese als Entwurf für ein optionales europäisches Versicherungsvertragsrecht ${ }^{12}$ ). Man wird kaum sagen können, dass schon heute eine lebendige europäische Wissenschaft des Versicherungsrechts existiert, aus der sich dieser Regeltext entwickelt hätte. Vielmehr wird ein optionales Instrument Ausgangspunkt einer europäischen Versicherungsrechtswissenschaft sein, die dann, so könnte man wiederum meinen ${ }^{13}$ ), in erster Linie dogmatisch arbeiten wird.

Doch warum hat sich die historische Rechtsvergleichung bisher nicht an der Schaffung einer europäischen Versicherungsrechtswissenschaft beteiligt? Ist eine historisch-vergleichende Bearbeitung des Versicherungsrechts nicht möglich oder nicht zielführend? Sie setzt gemeinsame historische Wurzeln der europäischen Versicherungsrechte oder zumindest Verknüpfungen zwischen den nationalen Entwicklung voraus.

\section{Nationale Forschungsstände}

Bei der Identifizierung gemeinsamer Wurzeln oder solcher Verknüpfungen stößt man indes sofort auf ein Problem. Die nationalen Geschichtsschreibungen stellen die Geschichte des Versicherungswesens und des Versicherungsrechts jeweils unterschiedlich dar und erwecken so den Eindruck, als würden gemeinsame Wurzeln oder Verknüpfungen nur eingeschränkt existieren.

Die deutschsprachige Literatur identifiziert drei Wurzeln des modernen

${ }^{9}$ ) Jürgen Basedow, Der Versicherungsbinnenmarkt und ein optionales europäisches Vertragsgesetz, in: Kontinuität und Wandel des Versicherungsrechts, FS Egon Lorenz, 2004, 93-110.

${ }^{10}$ ) Fritz Reichert-Facilides, Europäisches Versicherungsvertragsrecht?, in: FS Ulrich Drobnig, 1998, 119-134.

11) Jürgen Basedow et al. (Hgg.), Principles of European Insurance Contract Law, 2009.

${ }^{12}$ ) Statt vieler Helmut Heiss, Optionales Europäisches Versicherungsvertragsrecht, Rabels Zeitschrift für ausländisches und internationales Privatrecht [RabelsZ] 76 (2012), 317-338, 322, 335-338.

13) Hierzu siehe noch unten den Text nach Fn. 174. 
Versicherungswesens ${ }^{14}$ ). Die erste sei die Seeversicherung $\left.{ }^{15}\right)$. Sie sei Ursprung des kaufmännisch betriebenen Versicherungsgewerbes $\left.{ }^{16}\right)$. Über ihre Ursprünge, Entwicklung und Bedeutung besteht in der europäischen Forschung weitgehend Einigkeit: Sie entstand in Italien, und ihre Ausbreitung beschränkte sich zunächst auf den Mittelmeerraum; der Entstehungszeitpunkt wird zumeist in das 14. Jahrhundert gelegt ${ }^{17}$ ). Bis zum 16. Jahrhundert breitete sich die Seeversicherung dann von Italien und Portugal ausgehend über die Niederlande und Großbritannien nach Deutschland aus $\left.{ }^{18}\right)$. In den Niederlan-

$\left.{ }^{14}\right)$ Peter Koch, Versicherungswesen, in: Handwörterbuch zur deutschen Rechtsgeschichte [HRG] V, 1998, Sp. 815-826, 815; ders., Geschichte der Versicherungswirtschaft in Deutschland, 2012, 5; ders., Ansätze zum Versicherungsgedanken in deutschrechtlichen Quellen bis zur Hamburgischen Assekurenz- und Havereiordnung von 1731, in: Rechtsgeschichte und Rechtsdogmatik, FS Hermann Eichler, 1977, 367385, 367. Eine Bibliographie zur Geschichte des Versicherungsrechts findet sich bei Wilhelm Ebel, Quellennachweis und Bibliographie zur Geschichte des Versicherungsrechts in Deutschland, 1993, 1ff. Zu Österreich Wolfgang Rohrbach, Versicherungsgeschichte Österreichs von den Anfängen bis zum Börsenkrach des Jahres 1873, in: ders. (Hg.), Versicherungsgeschichte Österreichs, Bde. I-II 1988, hier I, 47-432.

$\left.{ }^{15}\right)$ Zur Seeversicherung vgl. vor allem die grundlegende Arbeit von Karin Nehlsen-von Stryk, Die venezianische Seeversicherung im 15. Jahrhundert, 1986.

${ }^{16}$ ) Statt vieler Koch, Geschichte (Fn. 14), 6.

${ }^{17}$ ) Vgl. Christoph M. Scheuren-Brandes, Insurance, in: Oxford International Encyclopedia of Legal History, Bd. III, 2009, 258; Giuseppe Stefani, Insurance in Venice from the Origins to the End of the Serenissima, Bd. I, 1958, 57ff.; Panay otis Perdikas, Die Entstehung der Versicherung im Mittelalter, 1966, passim; ders., Versicherung in den Dokumenten des Notars Rustico de Rusticis in Palermo, in: FS Reimer Schmidt, 1976, 325-353; Enrico Bensa, Il contratto di assicurazione nel medio evo, 1884, 46ff., 58ff.; Harold E. Raynes, A History of British Insurance, 2. Aufl. 1964, 5ff.; Florence Edler de Roover, Early Examples of Marine Insurance, Journal of Economic History 5 (1945), 172-200; Friedrich Plaß/Friedrich Robert Ehlers, Geschichte der Assecuranz und der hanseatischen Seeversicherungs-Börsen, 1902, 19; George Arnold Kiesselbach, Die wirtschafts- und rechtsgeschichtliche Entwicklung der Seeversicherung in Hamburg, 1901, 1ff.; Markus A. Denzel, Die Seeversicherung als kommerzielle Innovation im Mittelmeerraum und in Nordwesteuropa vom Mittelalter bis zum 18. Jahrhundert, in: Simonetta Cavaciocchi (Hg.), Ricchezza del mare - ricchezza dal mare, 2006, 574-609; Alberto Tenentini, Versicherung, in: Lexikon des Mittelalters [LexMA], Bd. VIII, 1997, Sp. 1582-1584; Bruno Dini, Seeversicherung, in: LexMA VII, 1995, Sp. 1691-1692; Richard Ehrenberg, Studien zur Entwickelungsgeschichte der Versicherung, Zeitschrift für die gesamte Versicherungswissenschaft [ZVersWiss] 1 (1901), 368-379.

${ }^{18}$ ) Vgl. im Detail mit Unterschieden Koch, Geschichte (Fn. 14), 42ff.; Thom as Dreyer, Die „Assecuranz- und Haverey-Ordnung“ der Freien und Hansestadt Ham- 
den und London war das Seeversicherungsgewerbe zunächst fest in der Hand italienischer Kaufleute, bevor es in London ab der Mitte des 16. Jahrhunderts von englischen Kaufleuten betrieben wurde ${ }^{19}$ ). In London ging damit einher, dass sich die Versicherer von den italienischen Gebräuchen ab- und den ebenfalls von ihren mediterranen Ursprüngen emanzipierten niederländischen Gebräuchen zuwendeten ${ }^{20}$ ). Der erste in Hamburg bezeugte Seeversicherungsvertrag datiert von $1588^{21}$ ). Anders als in London waren in Hamburg italienische Kaufleute im Seeversicherungsgewerbe niemals prägend, sondern von Beginn an orientierte man sich an den niederländischen Gebräuchen ${ }^{22}$ ). Im Übrigen war das Seeversicherungswesen immer in dem Sinne international, dass Versicherungsnehmer nicht zwingend mit heimischen Versicherern Verträge abschlossen ${ }^{23}$ ). Es handelt sich mithin um ein europäisches Phänomen, für dessen Erforschung sich ein vergleichender Zugriff geradezu aufdrängt,

burg von 1731, 1990, 25ff.; Raynes (Fn. 17), 17ff.; Barry Supple, The Royal Exchange Assurance, 1970, 6; Hugh A.L. Cockerell/Edwin Green, The British Insurance Business 1547-1970, 1976, 4; Violet Barbour, Marine Risks and Insurance in the Seventeenth Century, Journal of Economic and Business History 1 (1928), 561-596, 571; Charles Wrigth/C. Ernest Fayle, A History of Lloyd's, 1928, 34ff.; Winfried M. Hammacher, Die Grundzüge des allgemeinen Seeversicherungsrechts in der deutschen Gesetzgebung des 18. Jahrhunderts vor dem Hintergrund der älteren europäischen Seeversicherungsgesetzgebung, 1982, 17ff.; Ralph Neugebauer, Versicherungsrecht vor dem Versicherungsvertragsgesetz, 1990, 12; Denzel (Fn. 17), 592ff. Ein anderes Bild zeichnet Jean-Marie Hangartner, in: Handwörterbuch der Sozialwissenschaften, Bd. XI, 1961, 217-222, 218. Vgl. weiter Frederick Martin, The History of Lloyd's and of Marine Insurance in Great Britain, 1876, 6. Zur Ausbreitung in Frankreich Georges Hubrecht, Zur Geschichte der Versicherung in Frankreich, Versicherungswissenschaftliches Archiv [VersArch] 1958, 349-365, 352.

${ }^{19}$ ) David Ibbetson, Insurance, in: Oxford International Encyclopedia of Legal History III (Fn. 17), 252; S cheuren-Brandes (Fn. 17), 258; Raynes (Fn. 17), $22 \mathrm{ff.}$

${ }^{20}$ ) Guido Rossi, The Booke of Orders of Assurances, Maastricht Journal of European and Comparative Law [MJ] 19 (2012), 240-261.

$\left.{ }^{21}\right)$ Dreyer (Fn. 18), 25; Walther Heyn, Das schaffende Hamburg, 1950, 12; Kiesselbach (Fn. 17), 15; Peter Koch, 800 Jahre Hamburger Hafen, in: Heinz Leo Müller-Lutz/Karl-Heinz Rehnert (Hgg.), Beiträge zur Geschichte des deutschen Versicherungswesens, 1995, 268-284, 272; ders., Versicherungswesen (Fn. 14), Sp. 818; ders., Geschichte (Fn. 14), 44.

${ }^{22}$ ) Statt vieler Kiesselbach (Fn. 17), 109; Koch, Versicherungswesen (Fn. 14), Sp. 818. Zu den international sich gleichenden Bedingungen vgl. außerdem Siegbert Lammel, Rechtsbildung durch Verträge und Vertragsbedingungen, in: Karl Otto Scherner (Hg.), Modernisierung des Handelsrechts im 19. Jahrhundert, 1993, 89-117, 90ff.

23) Vgl. beispielhaft die Darstellung bei Raynes (Fn. 17), 97f. 
und dieser nicht auf nationale Entwicklungen beschränkte Ansatz ist schon bisher prägend.

Die übrigen Versicherungssparten etablierten sich in Europa vor allem im 18. und 19. Jahrhundert ${ }^{24}$ ). Ihre Ursprünge stellen sich in den verschiedenen europäischen Geschichtsschreibungen unterschiedlich dar. In der deutschen Forschung werden sie vornehmlich auf die beiden anderen Wurzeln des Versicherungswesens zurückgeführt: die durch die Gilden und Zünfte geleistete genossenschaftliche Absicherung ihrer Mitglieder vor allem bei Krankheit und Feuer ${ }^{25}$ ), aus denen die besonders bedeutsamen Brand-, Sterbe-, Witwenund Waisenkassen hervorgingen ${ }^{26}$ ), sowie die von Staats wegen betriebenen oder auf staatliche Initiative zurückgehenden Versicherungen ${ }^{27}$ ). Aus diesen beiden Wurzeln hätten sich vor allem die Lebens- und die Feuerversicherung

${ }^{24}$ ) Siehe die Darstellungen bei Neugebauer (Fn. 18), $13 f f$.

${ }^{25}$ ) Peter Koch, Entwicklung der versicherungsvertraglichen Rechtsquellen in Deutschland, in: FS Lorenz (Fn. 9), 411-426, 413; ders., Geschichte (Fn. 14), 15 ff.; ders., Der geistesgeschichtliche Hintergrund der Versicherungswirtschaft, in: Müller-Lutz/Rehnert (Fn. 21), 151-163, 153. Zur Absicherung durch die Gilden und Zünfte aus der umfangreichen Literatur Dieter Schewe, Geschichte der sozialen und privaten Versicherung im Mittelalter in den Gilden Europas, 2000; Frank Schulz-Nieswandt, Gilden als „totales soziales Phänomen“ im europäischen Mittelalter, 2000; Sigrid Fröhlich, Die Soziale Sicherung bei Zünften und Gesellenverbänden, 1974; Horst Peters, Die Geschichte der sozialen Versicherung, 3. Aufl. 1978, 21ff.; Volker Martens, Toten- und Knochenbruchgilden im Herzogtum Schleswig, 1967; Julius Kähler, Die Gilden in den holsteinischen Elbmarschen mit besonderer Berücksichtigung des Versicherungswesens, 1904, 71ff.; Wilhelm Schaefer, Urkundliche Beiträge und Forschung zur Geschichte der Feuerversicherung in Deutschland, Bd. I, 1911, 18ff.; Georg Helmer, Die Geschichte der privaten Feuerversicherung in den Herzogtümern Schleswig und Holstein, 2 Bd., 1925-1926.

${ }^{26}$ ) Ko ch, Geschichte (Fn. 14), 19, 26-29; ders., Geschichte der Versicherungswissenschaft in Deutschland, 1998, 44; Gerald Schöpfer, Sozialer Schutz im 16.18. Jahrhundert, 1976, 143ff.; Neugebauer (Fn. 18), 11, 13f.; Wilhelm Ebel, Die Professoren-Witwen- und -Waisenkassen, in: ders., Memorabilia Gottingensia, 1969, 73-100; Walter Fiedler, Die Geschichte des Versicherungswesens der Reichsstadt Nürnberg, 1958, 17ff. Zur Bedeutung dieser Kassen für die weitere Entwicklung Friedrich Wilhelm Ponfick, Geschichte der Sozialversicherung im Zeitalter der Aufklärung, 1940; J o a chim Gerla ch, Die Personenversicherung im Herzogtum Oldenburg bis zum Jahre 1876, 1967, 13ff.; Friedrich Edmund Horstmann, Versicherungseinrichtungen in der Stadt Hannover in der Zeit von 1728 bis 1885, 1965, 9ff.

${ }^{27}$ ) Koch, Versicherungswesen (Fn. 14), Sp. 816; ders., Geschichte (Fn. 14), 2942. Zur Geschichte der öffentlichen Feuerversicherung Georg Helmer, Entstehung und Entwicklung der öffentlich-rechtlichen Brandversicherungsanstalten in Deutschland, 1936 (ideologisch eingefärbt). 
entwickelt. Betont wird daneben noch die Bedeutung der Leibrente als eine frühe Form der Rentenversicherung ${ }^{28}$ ).

Zum Teil stellt die deutsche Literatur die genossenschaftliche Absicherung und die staatlichen Versicherungen nicht als zwei unterschiedliche Wurzeln des Versicherungswesens dar, sondern sie erweckt den Eindruck, dass sich die modernen Feuer- und Lebensversicherungen aus der genossenschaftlichen Absicherung entwickelt hätten und sie stellt die staatlichen Versicherungen als einen bloßen Zwischenschritt dieser Entwicklung dar ${ }^{29}$ ).

Spricht die deutsche Forschung von drei bzw. zwei Wurzeln des Versicherungswesens, so erweckt sie den Eindruck, dass die kaufmännisch betriebene Seeversicherung, die genossenschaftlich organisierte Absicherung durch Gilden und Zünfte sowie die staatlich betriebenen oder initiierten Versicherungen unabhängig voneinander entstanden seien und sich isoliert voneinander entwickelt hätten. Und in der Tat werden diese Entwicklungsstränge in der Literatur zumeist getrennt voneinander dargestellt.

Die englische Literatur zeichnet ein anderes Bild ${ }^{30}$ ): Sie unterstellt nicht, dass

${ }^{28}$ ) Vgl. Werner Ogris, Der Mittelalterliche Leibrentenvertrag, 1961, 113; der s., Leibrente, in: HRG II, 1978, Sp. 1800-1802; Ko ch, FS Eichler (Fn. 14), 378; Fiedler (Fn. 26), 3ff.; Schewe (Fn. 25), 154ff. Zur Leibrente vgl. zudem neuestens Hans-Georg Hermann, §§759-761, Leibrente, in: Joachim Rückert et al. (Hgg.), Historisch-kritischer Kommentar zum BGB, Bd. III, 2013.

${ }^{29}$ ) So wohl Sonja Heiss, Die Institutionalisierung der deutschen Lebensversicherung, 2006, 43; Koch, Versicherungswesen (Fn. 14), Sp. 816f. Ebenfalls von zwei Wurzeln sprechen: Wilhelm Ebel, Glücksvertrag und Versicherung, in: ders., Probleme der deutschen Rechtsgeschichte, 1978, 101-122, 101; Fanz Büchner, Geschichtliche Betrachtungen zum Begriff der Versicherung, in: Grundprobleme des Versicherungsrechts, FG Hans Möllers, 1972, 111-134, 112; ders ., Zum Begriff und zum Beginn der Versicherung, in: FS Karl Sieg, 1976, 111-125, 114; Walter Grosse, Versicherungsgeschichte, in: Handwörterbuch der Sozialwissenschaften, Bd. XI, 1961, 213-217, 213ff.; Hamburger Feuerkasse (Hg.), 1676-1951, 275 Jahre Hamburger Feuerkasse, 1951, 7.

${ }^{30}$ ) Zum Folgenden Ibbetson (Fn. 19), 253; Clive Trebilcock, Phoenix Assurance and the Development of British Insurance, Bd. I, 1985, 1ff.; Raynes (Fn. 17), passim; Terence O’Donnell, History of Life Insurance in its Formative Years, 1936, 78f.; Maurice Edward Ogborn, The Story of Life Assurance in the Experience of the Equitable Life Assurance Society, 1962, 17ff. Vgl. zudem Supple (Fn. 18), passim; Cockerell/Green (Fn. 18), 3ff., 34ff. In der deutschen Literatur ähnlich Hans Knoll, Aus der Entwicklungsgeschichte des Versicherungswesens von den Anfängen bis zur Gegenwart, 1934, 12; Lammel (Fn. 22), 96. Differenziert A. Fingland Jack, An Introduction to the History of Life Assurance, 1912, der zwar die Idee der Lebensversicherung in England in einen ähnlichen Kontext stellt wie die deutsche Literatur, aber auf 6 davon spricht, 
sich das Feuer- und Lebensversicherungswesen aus der genossenschaftlichen Absicherung durch Zünfte und Gilden sowie aus staatlich betriebenen oder initiierten Versicherungsanstalten entwickelt habe. Sie geht davon aus, dass die Lebensversicherung aus der Seeversicherung hervorgegangen sei, denn zunächst hätten vor allem Kaufleute die Leben von Schiffsmannschaften versichert, freilich nur für einzelne Reisen ${ }^{31}$ ). Die erste englische Lebensversicherungsgesellschaft auf versicherungsmathematischer Grundlage sei 1762 mit der Equitable Life Assurance Society gegründet worden ${ }^{32}$ ). Anlass für die Entstehung des Feuerversicherungswesens sei der Große Brand von London im Jahre 1666 gewesen $^{33}$ ). Das Recht der Feuerversicherung habe sich am See- und im weiteren Verlauf am Lebensversicherungsrecht orientiert. Englische Autoren gehen mithin nicht von zwei oder gar drei Wurzeln des Versicherungswesens aus, sondern von einer einzigen, und diese sehen sie in der Seeversicherung. Die übrigen Versicherungssparten sollen sich dann vor allem im 19. Jahrhundert und damit im Vergleich zum Kontinent zeitlich verzögert etabliert haben ${ }^{34}$ ).

Auch für die Niederlande und Flandern geht die Forschung nicht von getrennten Entwicklungssträngen aus, sondern meint, dass sich die Entwicklung insgesamt an der Seeversicherung orientiert habe ${ }^{35}$ ). Sie steht in der Forschung daher auch im Vordergrund ${ }^{36}$ ). Vereinzelt wird behauptet, dass es in Flandern bereits seit dem 13. Jahrhundert ein Feuerversicherungswesen gegeben habe ${ }^{37}$ ).

dass die modernen Lebensversicherung die Prinzipien der Seeversicherung adaptiert habe.

31) Vgl. Rossi (Fn. 20), 246f. Für Hamburg: Kiesselbach (Fn. 17), 124.

32) Monographisch zur Equitable Life Assurance Society Ogborn (Fn. 30).

33) Vgl. neben den in Fn. 30 genannten Fundstellen noch Raynes (Fn. 17), 74f.

${ }^{34}$ ) So Robin Pears on, Towards an historical model of services innovation, Economic History Review 50 (1997), 235-256.

${ }^{35}$ ) Vgl. J.P. van Niekerk, Sources of Insurance Law, in: Robert Feenstra/ Reinhard Zimmermann (Hgg.), Das römisch-holländische Recht, 1992, 305-327, 305ff.; S iegbert Lammel, Die Gesetzgebung des Handelsrechts, in: Helmut Coing (Hg.), Handbuch der Quellen und Literatur der Neueren Europäischen Privatrechtsgeschichte, Bd. II/2, 1976, 571-1083, 772ff.

${ }^{36}$ ) So bei J.P. van Niekerk, The Development of the Principles of Insurance Law in the Netherlands from 1500 to 1800, 2 Bde. 1998; ders., Sources (Fn. 35), 305ff. Vgl. zudem Sabine Go, Marine Insurance in the Netherlands 1600-1870, 2009; Frank C. Spooner, Risks at Sea, 1983; F. Kracht, Die Rotterdamer Seeversicherungs-Börse, 1922.

${ }^{37}$ ) So etwa von Knoll (Fn. 30), 9f.; Charles Farley Trenerry, The Origin and Early History of Insurance Including the Contract of Bottomery, 1926, $252 \mathrm{ff}$. 
Überwiegend nennt die Forschung aber auch hier das 17. Jahrhundert für die Etablierung der Feuerversicherung ${ }^{38}$ ).

In der französischen und italienischen Forschung steht die Seeversicherung ebenfalls im Zentrum des Interesses ${ }^{39}$ ). Dies mag darin einen Grund finden, dass etwa in Frankreich Lebensversicherungen durch die Ordonnance de la Marine von 1681 verboten waren ${ }^{40}$ ). Dagegen soll es in Italien, wie später in England, schon im Mittelalter üblich gewesen sein, dass im Zusammenhang mit Seeversicherungen auch Lebensversicherungen für die Schiffsmannschaft abgeschlossen wurden ${ }^{41}$ ). Noch vor London und Hamburg soll, so schreiben einige, die erste Feuerversicherung in Paris im Jahre 1666 gegründet worden sein $^{42}$ ). Andere erwähnen einen Brandentschädigungsverein aus dem Jahre $1545^{43}$ ). Meist wird die erste Feuerversicherung in Frankreich aber in das 18. Jahrhundert gelegt $\left.{ }^{44}\right)$. Schließlich gehen auch die italienische und französische Forschung nicht von mehreren Wurzeln des Versicherungswesens aus, sondern erwecken den Eindruck, als sei dessen Wurzel im Seeversicherungswesen zu suchen ${ }^{45}$ ). Daneben finden sich in Frankreich und Italien zwei Besonderheiten: die Tontinen und die soziale Versorgung durch die Montes Pietatis. Die Tontinen wurden durch den Italiener Lorenzo di Tonti (16301695) in der ersten Hälfte des 17. Jahrhunderts in Frankreich entwickelt $\left.{ }^{46}\right)$.

${ }^{38}$ ) Van Niekerk, Development (Fn. 36), I 424.

${ }^{39}$ ) Vgl. die Darstellungen bei Bensa (Fn. 17); Federigo Melis, Origine e sviluppi delle assicurazioni in Italia (secoli XIV-XVI), 1975; Hubrecht (Fn. 18), 349ff. Siehe auch Georges Hamon, Histoire générale de l'assurance en France et à l'étranger, 1897. Zur Entwicklung seit Ende des 18. Jahrhunderts in Frankreich vgl. André Straus, France, in: Peter Borscheid/Niels Viggo Haueter (Hgg.), World Insurance, 2012, 118-142, und in Italien vgl. Giandomenico Piluso, Italy, in: ebd., 167-188.

${ }^{40}$ ) Vgl. hierzu O’ Donnell (Fn. 30), 391; Hubrecht (Fn. 18), 356.

$\left.{ }^{41}\right)$ Vgl. den Hinweis bei Piluso (Fn. 39), 169.

42) Vgl. etwa Knoll (Fn. 30), 14.

${ }^{43}$ ) Schaefer, Bd. II (Fn. 25), 110.

${ }^{44}$ ) Vgl. mit unterschiedlichen Datierungen die Darstellungen bei Hubrecht (Fn. 18), 360; Hamon (Fn. 39), 32; Pierre Joseph Richard, Histoire des institutions d'assurance en France, 1956, 15; Straus (Fn. 39), 118.

$\left.{ }^{45}\right)$ Stefani (Fn. 17), 63, 119f.; Dini (Fn. 17), Sp. 1691; Hamon (Fn. 39); Richard (Fn. 44), 7ff.

$\left.{ }^{46}\right) \mathrm{Zu}$ den Tontinen vgl. Richard (Fn. 44), 25ff.; Albert Schug, Der Versicherungsgedanke und seine historischen Grundlagen, 2011, 245f.; Clemens v. Zedtwitz, Die rechtsgeschichtliche Entwicklung der Versicherung, 1999, 138ff.; Schöpfer (Fn. 26), 129ff.; Heinrich Braun, Geschichte der Lebensversicherung und der Lebensversicherungstechnik, 1925, 63ff.; Peter Koch, Zum Tontinengeschäft in versicherungshistorischer Sicht, in: ders., Beiträge zur Geschichte des deut- 
Die Montes Pietatis waren zunächst Kreditanstalten, entwickelten dann aber auch ein Versicherungsgeschäft $\mathrm{f}^{47}$ ).

\section{Mögliche Verknüpfungen der nationalen Entwicklungen}

Haben sich die Versicherungsrechte außerhalb der Seeversicherung also verschieden entwickelt, so dass eine historische Rechtsvergleichung mangels gemeinsamer Wurzeln oder Verknüpfungen zwischen den nationalen Entwicklungen wenig sinnvoll wäre? Ein solcher Schluss wäre voreilig.

So scheint die deutsche Literatur ein zeitbedingtes Auseinanderdenken verschiedener Entwicklungsstränge nur unreflektiert fortzuschreiben, wenn sie von zwei oder gar drei verschiedenen Wurzeln des Versicherungswesens spricht. So heißt es in einer Monographie aus dem Jahre 1936 ${ }^{48}$ ):

„Während also die Gegenseitigkeitsversicherung und die auf ihrer Grundlage aufgebaute öffentlich-rechtliche Brandversicherung eigenes, urdeutsches Gewächs ist, eine Schöpfung, auf die wir mit Recht stolz sein können, ist der Gedanke der Erwerbsversicherung vom Auslande her zu uns gekommen.“

Dem grundlegenden Werk von Viktor Ehrenberg aus dem Jahre 1893 ist diese Trennung unbekannt. Er schreibt ${ }^{49}$ ), „so hat sich auch der Versicherungsvertrag juristisch aus dem Seedarlehensvertrag entwickelt ${ }^{\star 50}$ ), und er stellt die Entwicklung damit ähnlich dar wie die englische Literatur. Levin Goldschmidt spricht von „zwei sich mannigfach verschlingende[n] Wurzeln“51). Nach ihm hat es zwar verschiedene Wurzeln des Versicherungswesens gegeben. Er behauptet aber nicht, dass sich im weiteren Verlauf getrennte Entwicklungsstränge unterscheiden ließen. Genau von dieser These geht die moderne deutsche Geschichtsschreibung aber

schen Versicherungswesens, Bd. II, 2005, 27-30; Julius Wyler, Die Tontinen in Frankreich, 1916, 6ff. Zu Lorenzo di Tonti Peter Koch, Pioniere des Versicherungsgedankens, 1968, 41ff.

${ }^{47}$ ) Zu den Montes Pietatis vgl. Schug (Fn. 46), 244f.; v. Zedtwitz (Fn. 46), 133ff.; Braun (Fn. 46), 43ff.

${ }^{48}$ ) Helmer (Fn. 27), 95. Ähnlich noch ders., Grundlinien der Geschichte der Versicherung, in: Internationales Versicherungsrecht, FS Albert Ehrenzweig, 1955, 57-70, 57ff.

${ }^{49}$ ) Victor Ehrenberg, Versicherungsrecht, 1893, 35. Vgl. auch Otto Hagen, Das Versicherungsrecht, in: Victor Ehrenberg (Hg.), Handbuch des gesamten Handelsrechts, Bd. VIII/1, 1922, 2ff.

50) Vgl. auch Schewe (Fn. 25), 28f. und Cornel Zwierlein, Der gezähmte Prometheus, 2011, 25, beide kritisch, wenn auch mit unterschiedlicher Stoßrichtung.

${ }^{51)}$ Levin Goldschmidt, Handbuch des Handelsrechts, Bd. I, 3. Aufl. 1891, 48. Zustimmend Büchner, FG Möller (Fn. 29), 112. 
regelmäßig aus, wenn sie diese Entwicklungsstränge isoliert voneinander untersucht.

Oft gehen die nationalen Geschichtsschreibungen von verschiedenen Versicherungsbegriffen aus ${ }^{52}$ ). Wer aber eine Geschichte der gewerblichen Versicherung schreibt, der blendet frühe Formen der genossenschaftlichen Absicherung aus ${ }^{53}$ ) und zeichnet ein anderes Bild als derjenige, der solche Formen einbezieht.

Schließlich wird in der deutschen Forschung eine regional verengte Perspektive und eine Fokussierung auf die Geschichte einzelner Akteure des Versicherungswesens geradezu zum Programm erhoben ${ }^{54}$ ):

„Die Versicherungsgeschichte ist [...] im Wesentlichen die Summe der Entwicklung zahlreicher einzelner Gesellschaften unterschiedlicher Größe und Rechtsform.“

So wichtig derartige Studien auch sind, gemeinsame Wurzeln der europäischen Versicherungsrechte oder auch nur Verknüpfungen zwischen den nationalen Entwicklungen vermögen sie kaum zutage zu fördern. Und außerhalb der Rechtsgeschichte wendet sich die Forschung in jüngerer Zeit Ansätzen zu, die diese regional verengte Perspektive abstreifen ${ }^{55}$ ).

1. Versicherungspraxis:

Schon in der modernen Literatur deuten sich vielfältige Verknüpfungen zwischen den Entwicklungen in den einzelnen europäischen Staaten an: Anlass für die Entstehung des modernen Feuerversicherungswesens in England soll der Große Brand von London im Jahre 1666 gewesen sein ${ }^{56}$ ). Aber genau diese Londoner Katastrophe soll auch ein Anstoß für die Gründung der Hamburger General-Feuer-Cassa im Jahre 1676 gewesen sein ${ }^{57}$ ), die erste staatlich betriebene Feuerkasse in Deutschland $\left.{ }^{58}\right)$.

52) Zum Problem der Fassung des Untersuchungsgegenstandes vgl. Friedrich Ebel, Rechtsgeschichtliche Entwicklung der Versicherung, in: Handwörterbuch der Versicherung, 1988, 617-626, 617; Bü chner, FG Möller (Fn. 29), 111ff.

${ }^{53}$ ) So die Beiträge in Borscheid/Haueter (Fn. 39). Siehe außerdem zur Geschichte der Lebensversicherung in den USA Sharon Ann Murphy, Investing in Life, 2010.

$\left.{ }^{54}\right)$ Koch, Geschichte (Fn. 14), 7. Diese regionale Fokussierung liegt auch ders ., Versicherungsplätze in Deutschland, 1986, zugrunde.

55) Vgl. Borscheid/Haueter (Fn. 39); Zwierlein (Fn. 50).

${ }^{56)}$ Siehe oben den Text zu Fn. 33.

${ }^{57}$ ) Scheuren-Brandes (Fn. 17), 257; Koch, FS Lorenz (Fn. 25), 417f.; ders ., Geschichte (Fn. 14), 29ff.; ders., Große Katastrophen der Versicherungsgeschichte, 1966, 33; Alexander Müssener, Die Entwicklung der Aachener Feuer-Versicherungs-Gesellschaft im 19. Jahrhundert, 2008, 25; Helmer (Fn. 27), 44, 55; Harald von Waldheim, Das Versicherungswesen in seiner Entwicklung, 1928, 10; Fritz 
Die Bedeutung der staatlichen Feuerkassen für die Entwicklung des Versicherungswesens in Deutschland ist unbestritten. Zwar wurden in England im Anschluss an den Großen Brand von London erste private Feuerversicherungsgesellschaften gegründet, aber fast zeitgleich mit der Hamburger Feuerkasse wollte auch die City of London 1681 eine Feuerversicherung gründen $^{59}$ ). Man möchte bei zwei identischen Vorstößen in Hamburg und London innerhalb von fünf Jahren nicht an einen Zufall glauben.

Englische, französische und belgische Lebens- und Feuerversicherer wirkten bis zur Mitte des 19. Jahrhunderts, zum Teil auch noch darüber hinaus ${ }^{60}$ ), in Deutschland ${ }^{61}$ ). Dabei verwendeten die englischen Versicherer ihre Versicherungsbedingungen auch in Deutschland, und die deutschen Versicherer lehnten sich zu Beginn des 19. Jahrhunderts an diese Bedingungen, die Feuerversicherer insbesondere an die der Phoenix Assurance Company, an ${ }^{62}$ ). Die englischsprachige Forschung geht zudem davon aus, dass die gewerblich betriebenen Lebensversicherungen in Frankreich unter dem Einfluss des englischen Lebensversicherungswesens standen ${ }^{63}$ ).

Morstein Marx, Zur Entstehung der Hamburger Feuerkasse, Hanseatische Rechtszeitschrift [HansRZ] 1927, Sp. 641-656, 652. Die Entwicklungen in Hamburg und London zeichnet zuletzt Z wi erlein (Fn. 50), 198ff., nach, der auf 226 in Frage stellt, die Hamburger Feuerkasse sei aus Anlass der Londoner Brands initiiert worden.

${ }^{58}$ ) Zu ihr Wilhelm Ebel, Die Hamburger Feuerkontrakte und die Anfänge des deutschen Feuerversicherungsrechts, 1936. Entgegen der vorherrschenden Ansicht will Knoll (Fn. 30), 12f., in der Königsberger Brandkonvention von 1627 die erste öffentlich-rechtliche Feuerversicherung erkennen. Dagegen Egid Fleck, Die Anfänge der öffentlich-rechtlichen Brandversicherung in den früheren Gebieten des heutigen Landes Baden-Württemberg, 1958, 6. Vorschläge zur Einrichtung einer öffentlichen Feuerversicherung soll es schon zu Beginn des 17. Jahrhunderts in Oldenburg gegeben haben: Schaefer (Fn. 43), 104, 115ff.; Koch, Pioniere (Fn. 46), 31ff.; Wilhelm Dursthoff, Die Entstehung, Entwickelung und Reform der oldenburgischen Brandkasse, 1904, 13ff.; Rudolf Bergmann, Geschichte des rheinischen Versicherungswesens bis zur Mitte des XIX. Jahrhunderts, 1928, 13. Kritisch zur Einordnung dieser Kassen in die Zweiteilung in öffentlich- und privatrechtlich Morstein Marx (Fn. 57), Sp. 641-656.

${ }^{59)}$ Hierzu Supple (Fn. 18), 7; Hangartner (Fn. 18), 218.

${ }^{60}$ ) Vgl. etwa Hans Menzel, Das englische Feuerversicherungsgeschäft in Deutschland und der Krieg, 1915, 3f.

$\left.{ }^{61}\right)$ Koch, FS Lorenz (Fn. 25), 423; ders., in: HRG V (Fn. 14), Sp. 819; ders., Geschichte (Fn. 14), 61; S. Heis s (Fn. 29), 39f.; Müs sener (Fn. 57), 32f.; Supple (Fn. 18), $156 \mathrm{f}$.

62) Koch, FS Lorenz (Fn. 25), 423f.; ders., Geschichte (Fn. 14), 49; Lammel, (Fn. 22), 103. Zur AGB-Praxis der Phoenix in Deutschland Trebilcock (Fn. 30), 194.

63) O’Donnell (Fn. 30), 391. 
In den Personen, die in Deutschland die ersten Lebensversicherungen auf den Weg gebracht haben, lassen sich weitere Verknüpfungen feststellen. Wilhelm Benecke (1776-1837), der 1806 die Hamburger Lebens-VersicherungsSocietät gründete, war Autor des in der ersten Hälfte des 19. Jahrhunderts führenden Lehrbuchs zum Versicherungsrecht und als solcher ein Kenner der englischen Versicherungspraxis und des englischen Versicherungsrechts ${ }^{64}$ ). 1823 unternahm Peter Willemsen in Elberfeld den Versuch, eine Lebensversicherung zu gründen. Zuvor war er Vertreter einer englischen Versicherung und damit wohl bestens vertraut mit der englischen Versicherungspraxis gewesen $^{65}$ ). Die Liste ließe sich fortsetzen ${ }^{66}$ ).

Das Gildewesen, dessen Bedeutung für die Entwicklung des Versicherungswesens in der deutschen Literatur unbestritten ist, ist selbstverständlich nicht auf Deutschland beschränkt gewesen, sondern erstreckte sich auf ganz Nordeuropa, und auch außerhalb Deutschlands hatten diese Gilden eine Sicherungsfunktion ${ }^{67}$ ). Für den Norden Frankreichs wird diese Absicherung durch Gilden ebenso wie in Deutschland als Vorläufer des Feuer- und Lebensversicherungswesens gesehen ${ }^{68}$ ).

Tontinen und die Montes Pietatis waren vor allem in Frankreich und Italien verbreitet, doch gab es sie auch in anderen europäischen Staaten ${ }^{69}$ ). Die

${ }^{64}$ ) S. Heiss (Fn. 29), 46; Koch, Geschichte (Fn. 14), 62. Zu Wilhelm Benecke vgl. auch Koch, Pioniere (Fn. 46), $217 \mathrm{ff.}$

${ }^{65)}$ S. Heiss (Fn. 29), 52.

${ }^{66}$ ) Vgl. S. Heiss (Fn. 29), passim. Vgl. zudem Denkschrift zum hundertjährigen Bestehen der Berlinischen Feuer-Versicherungs-Anstalt 1812-1912, 1912, 8. Eine Übersicht über die Gründungen auf dem Gebiet der Feuerversicherung gewährt Müssener (Fn. 57), 33ff. Vgl. schließlich noch Lammel (Fn. 22), 98.

${ }^{67}$ ) Vgl. Wilhelm Eduard Wilda, Das Gildewesen im Mittelalter, 1831, 123ff.; Paul Bütow, Die Versicherung auf Gegenseitigkeit, 1883, 10ff.; Schulz-Nieswandt (Fn. 25), 27ff.; S chewe (Fn. 25), passim; Hangartner (Fn. 18), 217f.; Hubrecht (Fn. 18), 359; Max Pappenheim, Ein altnorwegisches Schutzgildestatut, 1888; ders ., Die altdänischen Schutzgilden, 1885; Albert Rosin, Lebensversicherung und ihre geistesgeschichtlichen Grundlagen, 1932, 31ff.; Trenerry (Fn. 37), 246ff.; O’ D onnell (Fn. 30), 27ff., 61ff. Gilden, die ihre Mitgliedern bei Feuerschäden unterstützten, erwähnt auch Raynes (Fn. 17), 71.

${ }^{68}$ ) Vgl. beispielhaft die Darstellung bei Hubrecht (Fn. 18), 359.

${ }^{69}$ ) Scheuren-Brandes (Fn. 17), 258; S chöpfer (Fn. 26), 94ff., 132ff.; Braun (Fn. 46), 45ff., 63ff., 151ff.; O’ Donnell (Fn. 30), 163ff.; Werner Ogris, Tonti, Tontine, in: HRG V (Fn. 14), Sp. 276-277; Martina Spies, Feuerversicherung, Waisen- und Kreditkassen bei ostschwäbischen Reichsklöstern vor der Säkularisation und ihre Auflösung, 1997, 5; Koch, Tontinengeschäft (Fn. 46), 28f.; Fiedler (Fn. 26), 45ff.; Rohrbach (Fn. 14), I 138f. 
Tontinen fanden in Deutschland zudem Eingang in die Schriften der Kameralisten ${ }^{70}$ ) und sollen die Entwicklung der staatlichen Versicherungsanstalten ebenso wie die der Witwen- und Waisenkassen beeinflusst haben ${ }^{71}$ ). Witwen- und Waisenkassen sollen wiederum auch in Holland existiert haben $^{72}$ ). Schließlich ist die erste englische Lebensversicherungsgesellschaft zwar erst 1762 gegründet worden ${ }^{73}$ ), doch gab es bereits seit dem ausgehenden 17. Jahrhundert Vorläufer, deren Vordenker ein John Hartley (um 1700) war, der sich wiederum mit Tontinen beschäftigt hatte ${ }^{74}$ ).

Schließlich ist die Bedeutung des Leibrentenvertrags für die Entwicklung des Versicherungsrechts unbestritten ${ }^{75}$ ), er war auch in Frankreich und als life-annuities in England bekannt, Tontinen werden nur als Form des Leibrentenvertrags verstanden, und Leibrente, Witwen- und Waisenkassen sowie Tontinen sollen sich gegenseitig befruchtet haben ${ }^{76}$ ).

In der Forschung stellen sich diese Verknüpfungen als bloße Fußnoten einer im Kern nationalen Entwicklung $d \mathrm{r}^{77}$ ). Doch stellt sich die Frage, ob sie nicht so prägend waren, dass sich in ihnen gemeinsame Wurzeln der europäischen Versicherungsrechte finden ließen: Waren die ersten englischen Lebens- und Feuerversicherungen nicht europäisch, weil sie auf den europäischen Seeversicherungen beruhten? Wie europäisch waren die ersten englischen Lebensversicherungen, wenn bei ihrer Gründung die Tontinen eine Rolle spielten? Wie europäisch waren die staatlich betriebenen oder initiierten Versicherungen in Deutschland, wenn auch sie von den Tontinen beeinflusst waren? Wie englisch waren die deutschen Lebens- und Feuerversicherungen

${ }^{70}$ ) Vgl. Schöpfer (Fn. 26), 113ff.; S. Heiss (Fn. 29), 32ff.; Braun (Fn. 46), 43ff. Zur Bedeutung der Kameralisten für die Entwicklung des Versicherungswesens vgl. Hans Schmitt-Lermann, Der Versicherungsgedanke im deutschen Geistesleben des Barock und der Aufklärung, 1954, 33ff.; S chöpfer (Fn. 26), 25ff.; Wilhelm Hagena, Die Ansichten der deutschen Kameralisten des 18. Jahrhunderts über das Versicherungswesen, 1910; Peter Borscheid, Feuerversicherung und Kameralismus, Zeitschrift für Unternehmensgeschichte 30 (1985), 96-117.

${ }^{71}$ ) v. Zedtwitz (Fn. 46), 137; Braun (Fn. 46), 46ff.

$\left.{ }^{72}\right)$ Schöpfer (Fn. 26), 144.

$\left.{ }^{73}\right) \mathrm{Zu}$ ihr siehe bereits oben den Nachweis in Fn. 32.

${ }^{74}$ ) S. Heiss (Fn. 29), 39f.; Braun (Fn. 46), 113; Rosin (Fn. 67), 46ff. Zu John Hartley vgl. Koch, Pioniere (Fn. 46), 115ff. Vgl. außerdem Hangartner (Fn. 18), 219.

$\left.{ }^{75}\right)$ Siehe oben den Text zu und die Nachweise in Fn. 28.

${ }^{76}$ ) Vgl. nur Ogris (Fn. 28), 131; HKK-BGB/Hermann (Fn. 28), §§759-761 Rn. 20; Hubrecht (Fn. 18), 361; Rosin (Fn. 67), 35ff.

${ }^{77}$ ) Koch, Geschichte (Fn. 14), 6. Eine Ausnahme bildet etwa die Monographie von Braun (Fn. 46). 
in Deutschland zu Beginn des 19. Jahrhunderts, wenn die maßgeblichen Akteure auf ganz unterschiedliche Weise mit der englischen Versicherungspraxis in Kontakt gekommen waren? Diese Fragen können nur beantwortet werden, wenn die Praxis der Versicherer - also ihre Versicherungsbedingungen und ihre Statuten - bei einer historisch-vergleichenden Bearbeitung des Versicherungsrechts in den Blick genommen wird. Sie muss im Zentrum des Interesses stehen.

\section{Literatur:}

Mit Blick auf die Literatur drängen sich die Verknüpfungen zwischen den nationalen Entwicklungen nicht in gleicher Weise auf: Zwar gab es Schriftsteller, die in ganz Europa zitiert wurden. Dies gilt für einige Autoren des 16. Jahrhunderts, für Autoren, die sich mit der Seeversicherung befassten, und vereinzelt für Autoren des 19. Jahrhunderts ${ }^{78}$ ). Doch ist die Zahl der Schriften von europäischem Rang gering, und ihre Autoren beziehen zu vielen Einzelfragen oft keine Stellung. Zudem bildete sich eine breite versicherungsrechtliche Literatur nur langsam heraus.

\section{Gesetzgebung:}

Der Seeversicherung nahmen sich die Gesetzgeber früh an. Die entsprechenden Gesetze sind gut dokumentiert ${ }^{79}$ ). Die Forschung geht davon aus, dass die Gesetzgeber das in der europäischen Praxis geltende Seeversicherungsrecht nur einfingen und dass damit das europäische Seeversicherungsrecht in ihnen zum Ausdruck kommt, dass aber umgekehrt diese Gesetze auch auf die Praxis und die Gesetzgebung anderer Staaten ausstrahlten ${ }^{80}$ ).

Im Übrigen kristallisierte sich eine Gesetzgebung in Europa nur langsam heraus. In Deutschland kodifizierte das Allgemeine Landrecht für die Preußischen Staaten von 1794 schon früh das gesamte Versicherungsvertragsrecht $\left.{ }^{81}\right)$. Zudem existierten in den deutschen Staaten schon früh Nor-

${ }^{78}$ ) Vgl. Ehrenberg (Fn. 49), 46ff.; Koch, Geschichte (Fn. 26), 18ff., 76ff.; W. Ebel, Glücksvertrag (Fn. 29), 109ff.; F. Ebel, Entwicklung (Fn. 52), 620f.; Christoph Bergfeld, Die Stellungnahme der spanischen Spätscholastiker zum Versicherungsvertrag, in: Paolo Grossi (Hg.), La seconda scolastica nella formazione del diritto privato moderno, 1973, 457-471.

${ }^{79}$ ) Vgl. nur Lammel (Fn. 35), 571-1083, 608f., 683ff., 772ff., 824, 875ff., 908f., 962ff., 1017f., 1039f., 1067f.; F. Ebel, Entwicklung (Fn. 52), 617ff.; Koch, FS Lorenz (Fn. 25), 419ff.; Stefani (Fn. 17), 64; Hammacher (Fn. 18).

${ }^{80}$ ) Koch, Geschichte (Fn. 14), 45; Kiesselbach (Fn. 17), 131ff.

$\left.{ }^{81}\right)$ Vgl. nur Peter Koch, Die Behandlung des Versicherungsvertrages im preußischen Allgemeinen Landrecht, Versicherungsrecht 1994, 629-633. Zur weiteren Entwicklung ders., FS Lorenz (Fn. 25), 421f.; ders., Zur Geschichte der Versicherungsvertragsrechtlichen Kodifikationen in Deutschland und Österreich, in: FS R. Schmidt 
men zu den staatlichen Versicherungen ${ }^{82}$ ). Weiterhin bildeten sich seit Ende des 18. Jahrhunderts Normen zur Aufsicht über Versicherer heraus ${ }^{83}$ ). Dabei sollen die Gesetzgeber vergleichend gearbeite ${ }^{84}$ ) und sich an den Geschäftsbedingungen der Versicherer orientiert haben ${ }^{85}$ ). Wenn aber die Bedingungen der deutschen Versicherer unter dem Einfluss der ausländischen Praxis gestanden haben ${ }^{86}$ ), so hätte letztere auch die deutsche Gesetzgebung beeinflusst.

4. Rechtsprechung:

Weiterhin ist es möglich, dass die identifizierten Verknüpfungen auf die Rechtsprechung ausstrahlten: War die Praxis der Versicherer in Europa einheitlich oder doch ähnlich ${ }^{87}$ ), dann könnte sich dies auf die Rechtsprechung ausgewirkt haben. Denn dann könnte sich die Argumentation der Versicherer auf Grundlage dieser Versicherungspraxis vor den nationalen Gerichten ebenfalls auf vergleichbaren Bahnen bewegt haben.

Freilich ist dabei zu beachten, dass die Rechtsprechung zum Versicherungsrecht bisher kaum aufgearbeitet ist. Sie entwickelte sich in den einzelnen

(Fn. 17), 299-323, 303ff.; Neugebauer (Fn. 18), 28ff.; Angela Duvinage, Die Vorgeschichte und die Entstehung des Gesetzes über den Versicherungsvertrag, 1987, $3 \mathrm{ff}$.

${ }^{82}$ ) Vgl. W. Ebel, Quellennachweis (Fn. 14), 149ff.; Lammel (Fn. 35), 614f., $712 \mathrm{ff}$.

83) Vgl. Michael Tigges, Geschichte und Entwicklung der Versicherungsaufsicht, 1985; Peter Koch, Der Weg zur einheitlichen Staatsaufsicht über Versicherungsunternehmen in Deutschland, in: Bundesaufsichtsamt für das Versicherungswesen (Hg.), 100 Jahre materielle Versicherungsaufsicht in Deutschland, Bd. I, 2001, 5-24; ders., Geschichte (Fn. 14), 25; Georg Heinrich Maurer, Über die historische Entwicklung der Versicherungs-Aufsicht in Deutschland, 1911; Hans Christoph Atzpodien, Die Entwicklung der preußischen Staatsaufsicht über das private Versicherungswesen im 19. Jahrhundert unter besonderer Berücksichtigung ihres Verhältnisses zum Wirtschaftsliberalismus, 1982.

${ }^{84}$ ) Vgl. Koch, Weg (Fn. 83), 16ff.; Werner Ogris, Zur Entwicklung des Versicherungsaufsichtsrechts und des Versicherungsvertragsrechts in Österreich von der Mitte des 19. Jahrhunderts bis zum Ende der Monarchie, in: Rohrbach (Fn. 14), II 1-152, 16f. Zu französische Vorbildern zu Beginn des 19. Jahrhunderts in Hamburg Hans Peter Ipsen, Zur Entwicklung der Versicherungsaufsicht in Hamburg, Hanseatische Rechts- und Gerichts-Zeitschrift 1939, Sp. 89-118; ders., Juristische Hamburgensien, in: Heinrich Ackermann et al. (Hgg.), Aus dem Hamburger Rechtsleben, 1979, 249-290, 277f. Vgl. zudem Heyn (Fn. 21), 7; Neugebauer (Fn. 18), 52, 56.

${ }^{85}$ ) Vgl. wiederum das Beispiel bei Neugebauer (Fn. 18), 56.

${ }^{86)}$ Siehe dazu oben den Text zu und die Nachweise in Fn. 60-66.

${ }^{87}$ ) Dazu oben der Text zu Fn. 60-63. 
Staaten wohl zeitlich verzögert: In England, so die moderne Forschung, spiegelte sie bis in die zweite Hälfte des 18. Jahrhunderts nur die Praxis der Versicherer wider, bevor Lord Mansfield (1705-1793) in den 1760er bis 1780er Jahren die Grundlagen des modernen Versicherungsrechts legte und sich im 19. Jahrhundert eine verfeinerte Rechtsprechung entwickelte ${ }^{88}$ ). In Deutschland hat sich eine Rechtsprechung wohl erst später herausgebildet, wurden doch die ersten kaufmännisch betriebenen Feuer- und Lebensversicherungen zu Beginn des 19. Jahrhunderts überhaupt erst gegründet. Zudem ist zu berücksichtigen, dass etwa im Feuerversicherungswesen in England und Deutschland zunächst Schiedsklauseln weite Verbreitung fanden oder dass der Instanzenzug zum Teil verkürzt war, so dass sich (höchstrichterliche) Rechtsprechung kaum entwickeln konnte ${ }^{89}$ ). Freilich lassen sich über die Entwicklung der Rechtsprechung zum Versicherungsrecht in Deutschland kaum belastbare Aussagen treffen, hat die moderne Forschung sie doch bisher erst für den Zeitraum ab dem ausgehenden 19. Jahrhundert untersucht ${ }^{90}$ ).

5. Allgemeines Vertragsrecht:

Lässt man die staatlich betriebenen Versicherungen außen vor, so lagen Versicherungen immer Verträge zugrunde, und der Versicherungsvertrag gehört in die allgemeine Vertragslehre. Dass aber die nationalen Vertragsrechte gemeineuropäische Wurzeln aufweisen, ist unbestritten. Das allgemeine Vertragsrecht als dogmatische Grundlage des Versicherungsvertragsrechts mag mithin dazu geführt haben, dass identische Konflikte mit den identischen Rechtsfiguren des allgemeinen Vertragsrechts gelöst worden sind. Auch hieraus können sich Verknüpfungen zwischen den Entwicklungen in den einzelnen europäischen Staaten ergeben.

\section{Drei Beispiele einer historisch-vergleichenden Bearbeitung des Versicherungsrechts}

Eine historisch-vergleichende Bearbeitung des Versicherungsrechts scheint also möglich. Aber lohnt sie sich auch? Dieser Frage soll anhand von drei Beispielen nachgegangen werden. Dem bisher Gesagten folgend soll die Praxis der Versicherer im Mittelpunkt stehen und beleuchtet werden, wie die Rechtsprechung auf diese Praxis reagiert hat. Die Beispiele sind auf einen Vergleich zwischen England und Deutschland beschränkt. Denn auch im Versicherungs-

${ }^{88}$ ) Ibbets on (Fn. 19), 252.

${ }^{89}$ ) Vgl. hierzu etwa Müss ener (Fn. 57), 139.

${ }^{90}$ ) Vgl. etwa Tobias Prang, Der Schutz der Versicherungsnehmer bei der Auslegung von Versicherungsbedingungen durch das Reichsgericht, 2003. 
recht gehen viele von nur schwer überwindbaren Unterschieden zwischen den kontinentaleuropäischen Rechten und dem englischen Recht aus ${ }^{91}$ ), und sehen diese als historisch bedingt $\mathrm{an}^{92}$ ).

1. Verhaltensanforderungen im Lebensversicherungswesen:

Versicherer müssen bei Abschluss einer Lebensversicherung in der Lage sein, das vertraglich zu übernehmende Risiko einzuschätzen. Dafür muss der Versicherungsnehmer risikobeeinflussende Umstände offenbaren, etwa Vorerkrankungen. Im geltenden deutschen Recht spricht man von Obliegenheiten $^{93}$ ). Im Folgenden soll die Entwicklung dieser Verhaltensanforderungen in den Statuten und Bedingungen der Lebensversicherungen und ihrer Vorläufer in England und Deutschland nachverfolgt werden.

a) Entwicklung in England:

Die erste englische Lebensversicherungsgesellschaft auf fester versicherungsmathematischer Grundlage ist mit der Equitable Life Assurance Society 1762 gegründet worden ${ }^{94}$ ). Doch hatte es Vorläufer gegeben ${ }^{95}$ ), so die Amicable Society aus dem Jahr 1706 ${ }^{96}$ ). Daneben existierten in England Witwenund Waisenkassen, deren Bedeutung für die Entwicklung des deutschen Lebensversicherungsrechts immer wieder betont wird ${ }^{97}$ ). Und schon früh finden sich in den Bedingungen einer solchen Kasse entsprechende Verhaltensanforderungen. Die Bedingungen der Assurance for Widows and Orphans aus dem Jahre 1699 bestimmten ${ }^{98}$ ):

„7. That every Subscriber shall, within Six Months after his Subscribing these Presents, appear before Three or more of the Trustees, at their Court to be held for

$\left.{ }^{91}\right)$ Jürgen Basedow, The Optional Application of the Principles of European Insurance Contract Law, ERA Forum 2008, 111-117, 111. Vgl. auch Fritz ReichertFacilides, Rechtsvereinheitlichung oder Rechtsvielfalt?, in: Fritz Schwind (Hg.), Europarecht - Internationales Privatrecht - Rechtsvergleichung, 1988, 155-170, $159 \mathrm{ff}$.

${ }^{92}$ ) Heinrich Frommknecht, Gibt es eine westfälisch-lippische Versicherungsgeschichte?, in: Peter Koch, Geschichte der westfälisch-lippischen Versicherungswirtschaft und ihrer Unternehmen, 2005, 7-11, 7.

${ }^{93}$ ) Siehe nur Manfred Wandt, Versicherungsrecht, 5. Aufl. 2010, Rn. 1183ff., 782ff. Rechtsvergleichend Giesela Rühl, Obliegenheiten im Versicherungsvertragsrecht, 2004.

$\left.{ }^{94}\right)$ Siehe oben den Text zu und die Nachweise in Fn. 32.

95) Siehe oben den Text zu und die Nachweise in Fn. 74.

$\left.{ }^{96}\right)$ Lammel (Fn. 35), 1074.

${ }^{97}$ ) Siehe oben den Text zu und die Nachweise in Fn. 26.

${ }^{98}$ ) Zitiert aus David Jenkins/Takau Yoneyama (Hgg.), History of Insurance, Bde. I und III, 2000, hier III, 312. Zu dieser Kasse vgl. Braun (Fn. 46), 113; Rosin (Fn. 67), 46. 
this Society, to have his Policy Approv'd; and shall, if requir'd by the Trustees, or Master of the Office, produce a Certificate of his Age, as also an Affidavit, that he believes himself to be under the Age of Fifty, and in good state of Health. [...].“

Der Versicherungsnehmer musste sein Alter nachweisen sowie, dass er in gutem Gesundheitszustand ist. Detailliertere Angaben zum Gesundheitszustand oder weitere Informationen zu anderen risikorelevanten Faktoren scheinen noch ebenso wenig erforderlich gewesen zu sein wie ärztliche Untersuchungen. Vor allem aber schwiegen die Bedingungen zu den Rechtsfolgen eines Verstoßes gegen diese Verhaltensanforderungen.

Andere Bedingungen der Zeit waren sogar noch unbestimmter, als sie es nur zu einer Bedingung des Versicherungsschutzes erklärten, dass der Versicherungsnehmer ein bestimmtes Alter hat und gesund ist, aber keine Verhaltensanforderung formulierten, nach der sich der Versicherungsnehmer zu beiden Umständen erklären musste. So stellte der Plan für die Errichtung einer Friendly Society for Insuring Lives von circa 1700 schlicht fest ${ }^{99}$ ):

„III. That the Person or Persons for whose Life or Lives any one subscribes, shall be healthful, and be above the Age of Twelve Years, and under the Age of Fifty.“

In der Folgezeit lassen sich mit Blick auf alle Aspekte - die Zahl der zu offenbarenden risikobeeinflussenden Umstände, die ausdrückliche Formulierung von Verhaltensanforderungen und die Bestimmung der Rechtsfolgen - Fortschritte erkennen. So bestimmten die Versicherungsbedingungen der Equitable Life Assurances Society aus dem Jahre $1762^{100}$ ):

„IV. Every person desiring to make assurance with the Society, must sign a Declaration, setting forth the age, state of health, profession, occupation, and other circumstances of the persons, whose lives are proposed to be assured. This declaration is the basis of the contract between the Society and the person desiring to make such assurance; and if any artful, false, or fraudulent representation shall be used therein, all claim on account of any policy so fraudulently obtained shall cease, determine, and be void.“

Im ersten Satz werden die Verhaltensanforderungen auf den Beruf und, indes sehr unbestimmt, auf andere Umstände erstreckt. Und bei fehlerhaften Angaben sollen Ansprüche aus dem Versicherungsvertrag erlöschen. Freilich führte ein erhöhtes Risiko, wenn es offenbart worden war, nicht zwingend dazu, dass der Versicherer dem Versicherungsnehmer den Vertragsschluss verwehrte. Vielmehr konnte der Versicherer auch einen Risikozuschlag verlangen, wie die gleichen Bedingungen verdeutlichen ${ }^{101}$ ):

${ }^{99}$ ) Zitiert aus Jenkins/Yoneyama (Fn. 98), III 316.

${ }^{100}$ ) Zitiert aus Jenkins/Yoneyama (Fn. 98), III 381.

${ }^{101}$ ) Zitiert aus Jenkins/Yoneyama (Fn. 98), III 380. 
„III. [...] The lives [...] of Men whose occupations are hazardous, may be assured upon payment of such advance in the premiums as shall be judged adequate to the hazard.“

Die Rechtsfolgen der Versicherungsbedingungen des gleichen Versicherers wurden sodann bis zum Jahre 1795 noch einmal dahin konkretisiert, dass auch die bereits durch den Versicherungsnehmer gezahlten Prämien bei einem Verstoß gegen die Verhaltensanforderungen verfielen ${ }^{102}$ ):

„Every person desirous to make assurance with the society must sign a declaration by himself or agent, setting forth the age, state of health, profession, occupation, and other circumstances of the persons whose lives are proposed to be assured [...]. This declaration is the basis of the contract between the society and the person desirous to make such assurance; and if any artful, false, or fraudulent representation shall be used therein, all claim on account of any policy so obtained, shall cease, determine, and be void, and the monies which shall have been paid upon account of such assurance, shall be forfeited to the use of the society.“

In der gedruckten Erklärung der Rock Life Assurance Company aus dem Jahre 1809, die der Versicherungsnehmer auszufüllen hatte, fällt auf, dass nicht nur allgemein nach dem Gesundheitszustand, sondern auch nach besonderen Krankheiten gefragt wurde ${ }^{103}$ ):

„I ... by profession or occupation, ... born in the parish of ... in the county of ... in the month of ... in the year ... and now resident at ... in the ... county of ... being desirous of making an Assurance for the term of ... on my own Life, to the amount of $£$... with the Rock Life Assurance Company: DO DECLARE, that my Age does not exceed ... years; that I have ... had the small pox and ... the gout; that I have ... had a rupture; have ... suffered a spitting of blood; ... and that I am not afflicted with any disorder tending to shorten life; and I do hereby agree that this DECLARATION be the ground of the Contract between me and the said Company, and that if it be not true, all money paid to the Society by me, on account of the Assurance, shall be forfeited.“

Im Jahre 1809 offenbart sich in den Bedingungen der Provident Institution for the Insurance of Lives and the Grant and Purchase of Annuities eine weitere Neuerung. Die bloße Erklärung durch den Versicherungsnehmer war nicht ausreichend. Er musste einen Arzt als Referenz benennen ${ }^{104}$ ):

${ }^{102}$ ) A short account of the Society for Equitable Assurances on Lives and Survivorships established by deed Inrolled in his Majesty's Court of Kings' Bench at Westminster, 1795, 9f.

103) The principles of life assurance explained: together with new plans of assurance and annuities adapted to the prudent of all classes civil and military by the Rock Life Assurance Company, 1809, 45.

${ }^{104}$ ) A Short Account of the Provident Institution, specially empowered by Act of Parliament for the Insurance of Lives, and the Grant and Purchase of Annuities, 1809. 
„Persons desiring insurance on lives, or on survivorship, must state the name, residence, and occupation of the party, on whose life the insurance is proposed to be made, the precise age of such party, the place of birth, whether employed in any military or naval service, and whether afflicted with any ailment tending to the shortening of life; they must give a reference to some medical or other person whose evidence may be satisfactory on these points: - They must also sign a declaration, verifying the above particulars, forming the basis of the contract between the insured and this Institution, in which, if any artful, false, or fraudulent representation shall be used, all claim, on account of any policy so obtained, shall cease, determine, and be void, and the monies which shall have been paid upon account of such insurance, shall be forfeited to the use of the Institution.“

In den Bedingungen der Albion Fire and Life Insurance Company aus dem Jahre 1809 fällt schließlich auf, dass die Versicherer fast wortgleiche Klauseln verwendeten, und das deutet auf Vereinheitlichungsbestrebungen in den Allgemeinen Versicherungsbedingungen hin ${ }^{105}$ ):

„Persons desiring Insurance on Lives, or on Survivorship, must state the name, residence, and occupation of the party, or parties, on whose Life, or Lives, the Insurance is proposed to be made; the precise age of such party, or parties; their place of birth; whether employed in any military or naval service; and whether subject to Gout, Asthma, or other ailment, tending in any degree to the shortening of life. They must give a reference to some medical or other person, whose evidence may be satisfactory on these points. If the Insurance be undertaken, they must sign a declaration of the several facts stated, which declaration shall be taken as the basis of the contract between the assured and the Company; and the Insurance shall be valid only in the case of such declaration being found to contain, In all respects, a true and faithful disclosure of every circumstance to which it may purport to have reference.“

Die gleichen Bedingungen zeigen auf, wie der Versicherungsnehmer vorzugehen hatte, wollte er eine Versicherung abschließen: Er musste die Erklärungen schriftlich abgeben und persönlich erscheinen. Ein Erscheinen war schon nach den Bedingungen der Assurance for Widows and Orphans erforderlich gewesen $^{106}$ ). Konnte er nicht persönlich erscheinen, musste er ein Attest, etwa eines Arztes, bestärkt durch Leumundszeugnisse vorlegen. Einen Wortlaut des Attestes und des Leumundszeugnisses gab die Albion Fire and Life Insurance Company wie folgt vor ${ }^{107}$ ):

„We, A D, and C D, Physicians, or Surgeons, or Apothecaries, residing in the city of Gloucester, having considered the above proposition for an Insurance of 1,000l. on the Life of Edward Johnson, do hereby certify, that we are acquainted with the state

105) Proposals from the Albion Fire and Life Insurance Company, of London, instituted 1805; and empowered by Act of Parliament, 1809.

${ }^{106)}$ Siehe oben das Zitat zu Fn. 98.

${ }^{107)}$ Proposals (Fn. 105). 
of health and with habits of life, or that we have [...] enquired into the state of health and habits of life, of the said Edward Johnson; and we declare it to be our opinion and belief, that the said Edward Johnson seems present in a sound state of body, and that he is not subject to any disease, ailment, or infirmity, now accustomed to habits, which tend to shortening of life, or to the prejudice of health.

And I, E F, Merchant, residing in the said city of Gloucester, do hereby declare, that I am and long have been, well acquainted with the said Edward Johnson; and [...], in my opinion and belief, that the said Edward Johnson is in all respects a fair and safe subject for Life Insurance; and that judgment above made by A B, and C $\mathrm{D}$, has been founded on actual knowledge, or on a [...] faithful investigation, and contains no averment contrary to truth.“

„I, G H, Mayor of Gloucester, and we, I K, L M, and N O, the Minister and Churchwardens of the Parish of ... in the City, or County of ... do certify that I, or we, know A B, C D, and E F, the several parties who are subscribers to the certificate, or declaration hereunto prefixed; that the said A B, C D, and E F, are persons of good character, and competent judgment; and that in my, or our, opinion and belief that any certificate or declaration so subscribed, it entitled to full credit and authority.“

\section{b) Entwicklung in Deutschland:}

In Deutschland sind Lebensversicherungen im modernen Sinne seit dem 19. Jahrhundert gegründet worden ${ }^{108}$ ). Doch hatte es Vorläufer gegeben: die erwähnten Sterbe-, Witwen- und Waisenkassen ${ }^{109}$ ). Viele dieser Kassen wurden staatlich betrieben oder gingen auf staatliche Initiative zurück, und es bestand eine Versicherungspflicht. Bei Pflichtversicherungen trat das untersuchte Regelungsproblem nicht auf. Auch in den Satzungen von Gilden finden sich entsprechende Vorschriften nicht ${ }^{110}$ ). Doch gab es auch schon früh freiwillige Kassen. Ihre Bedingungen entwickelten sich ähnlich wie die der englischen Versicherer. Die Statuten einer Sterbe-Kasse in Berlin aus dem Jahre 1710 bestimmten ${ }^{111}$ ):

„Art. I. Von der Eigenschafft derer Interessenten / und ihrem Alter. Derjenige / so ein Mit-Glied dieser Societät werden will / soll sich mit einer ehrlichen Profession und Beruffs-Arbeit nähren / auch eine gute Nachrede eines ehrbaren Wandels und Christlichen Lebens haben [...]; So soll auch / bey Anfang dieser Cassa, keiner / so über 56. Jahr alt ist / eingenommen werden / es sey männlichs oder weibliches Geschlechts; dafern aber jemand so höheren Alters / mit eingeschrieben seyn wolte / so soll derselbe gleich bey der Angabe / jegliches Jahr / als älter er ist / mit einem Thaler vergüten. [...].“

${ }^{108)}$ Siehe oben den Absatz zu Fn. 64.

${ }^{109}$ ) Zu ihnen siehe oben den Text zu und die Nachweise in Fn. 26.

${ }^{110}$ ) Vgl. beispielhaft die Satzung der Wandsbeker Kranken- und Totenlade von 1677, abgedruckt in A. Möller, Die älteste Wandsbeker Kranken- und Totenlade, Zeitschrift für Versicherungswesen 1952, 343-344.

${ }^{111}$ ) Zitiert aus Schöpfer (Fn. 26), 214. 
Auch in Deutschland war mit Beruf, Alter und Lebenswandel zunächst nur eine eingeschränkte Zahl von Faktoren bestimmend, und es begegnet früh die Idee eines Zuschlags bei älteren Versicherungsnehmern. Art. I formuliert, wie die frühen englischen Statuten, keine Verhaltensanforderungen, sondern nur Bedingungen der Aufnahme in die Kasse. Dass damit Verhaltensanforderungen verbunden waren, offenbart Art. V, der die Rechtsfolgen ihrer Verletzung festlegt ${ }^{112}$ ):

„Art. V. Wie [...] diejenige zu bestraffen / so ihr rechtes Alter verschweigen. [...] Es sollen auch diejenigen / so dem Cassa-Register wollen einverleibet werden / bey dem ältesten Vorsteher erscheinen / damit er sehen könne / ob sie noch frisch und gesund seyn / und also die sämtliche Interessenten mit allzu zeitiger Aufflage nicht mögen beschweret werden / welcher aber sein rechtes Alter nicht angezeigt / und also beym einschreiben schon über obgenannte Jahr alt ist / bey dessen Absterben soll zur Straffe 10. Thlr. weniger als andern gezahlet werden / welches Geld laut Art. 12 und 13. soll angewandt werden.“

Das Verfahren, das ein Versicherungsnehmer durchlaufen musste, schimmerte bereits in den englischen Bedingungen durch: Er musste vor der Kasse erscheinen. Doch anders als die zeitgleichen englischen Bedingungen bestimmten die Statuten dieser Kasse bereits die Rechtsfolge der Verletzung der Verhaltensanforderung. Freilich war diese milde: Der Begünstigte verlor nicht seinen Anspruch aus dem Versicherungsverhältnis, sondern musste nur einen Abschlag bei der Leistung des Versicherers hinnehmen. Indes wurde in Deutschland schon bald eine andere Rechtsfolge üblich, wie die Herzoglich-Würtembergische Ordnung für die allgemeine freywillige Wittwen- und Waysen-Cassa von 1756 verdeutlicht ${ }^{113}$ ):

„§10. Wer in diese Gesellschafft eintretten will, muß beybringen: 1. Einen Original-Tauff-Schein, oder anderen eben so gültigen Beweis, wann er gebohren seye; so dann 2. Ein Attestat von seinem ordentlichen Medico, oder der Obrigkeit seines Orts, daß er noch bey guter Gesundheit seye: Sollte sich aber nachhero äußeren, daß ein solches Attestat nicht der Wahrheit gemäß gewesen; ist nicht nur der Einleger seiner Einlage und der Gesellschafft verlustig, sondern es werden auch Seine Hoch-Fürstliche Durchlaucht dergleichen Ungebühr auf unterthänigste Anzeige nachdrücklich ahnden.“

Genau wie die Bedingungen der englischen Versicherer bestimmte diese Ordnung, dass der Versicherungsnehmer seine Ansprüche aus der Kasse verlor, wenn die Angaben falsch waren. Und weiterhin deutet sich an, dass auch in Deutschland Atteste durch Ärzte üblich wurden.

\footnotetext{
$\left.{ }^{112}\right)$ Zitiert aus Schöpfer (Fn. 26), 216.

113) Herzoglich-Würtembergische Ordnung für die allgemeine freywillige Wittwen- und Waysen-Cassa von 1756, 1756, 5.
} 
Schließlich gingen auch die deutschen Kassen dazu über, nach bestimmten Erkrankungen zu fragen, und sie verlangten, dass das ärztliche Attest bestärkt wurde. Beides wird im Reglement der 1775 gegründeten Königlich Preußischen Allgemeinen Witwen-Verpflegungs-Anstalt deutlich ${ }^{114}$ ):

„§3. Dergleichen gänzlich ausgeschlossene Personen sind: a) Männer über Sechzig Jahre, b) Seefahrer von Metier, c) Männer, welche mit Schwindsucht, Wassersucht, oder einem andern morbo chronico behaftet sind, der einen nahen Tod befürchten läßt. [...].

$\S 7$. Es hat also zuförderst ein jeder der Theil nehmen will, in Ansehung des Alters, für sich [...] einen Taufschein beyzubringen. [...].

§9. Endlich muß er ein Attest eines approbirten Medici Practici beybringen, worinn derselbe auf seine Pflicht und an Eides-Statt versichert, daß nach seiner besten Wissenschaft, der Recipiendus weder mit der Schwindsucht, Wassersucht, noch einem andern Morbo chronico, so ein baldiges Absterben befürchten ließe, behaftet, auch überhaupt zur Zeit nicht krank noch bettlägerig, sondern gesund, nach Verhältnis seines Alters bey Kräften, und fähig sey seine Geschäfte zu verrichten. Dieses Attest des Medici muß von Vier Mitgliedern Wittwen-Societät, oder wenn solche nicht zu haben sind, von Vier andern bekannten redlichen Männern unterschrieben werden, welche bezeugen: daß ihnen der Recipiendus bekannt sey, und sie das Gegentheil von dem, was der Medicus attestiret, nicht wissen. [...].

$\S 13$. Wir versprechen Uns, daß in Ansehung dieser Atteste, sowohl diejenigen, welche derselben benöthiget sind, als diejenigen, welche sie ausstellen müssen, mit der strengsten Redlichkeit verfahren werden. Damit jedoch hierunter aller Betrug, welcher dieser gemeinnützigen Anstallt zum größten Nachtheil gereichen könnte, gänzlich vermieden [...] werden möge: So setzten Wir hiermit fest und verordnen, daß [...] diejenigen Unserer Unterthanen, welche hierunter eines falsi überführet werden können, [..] die Recipirten selbst [...], wenn ein Betrug hierunter zu irgend einer Zeit entdeckt, und durch den Ausspruch des ordentlichen Richters des Beschuldigten als erwiesen erkannt wird, den Verlust ihrer eingelegten Gelder und dadurch erhaltenen Rechte, ohnfehlbar zu gewärtigen haben sollen.“

In der ersten Hälfte des 19. Jahrhunderts lässt sich wie in England eine Konsolidierung der Bedingungen ausmachen. Nahezu alle Versicherer verlangten eine umfassende Deklaration, stellten dafür ein Formular zur Verfügung, verlangten ein ärztliches Attest, erforderten dabei die Erklärung zu bestimmten Krankheiten und legten Wert auf Leumundszeugnisse ${ }^{115}$ ). Auch

${ }^{114}$ ) Patent und Reglement für die Königlich Preußische allgemeine WitwenVerpflegungs-Anstalt, Novum Corpus Constitutionum Prussico-Brandenburgensium Praecipue Marchicarum, Bd. V/E, Sp. 383-387.

$\left.{ }^{115}\right)$ Vgl. §§ 42f. der Verfassung der Lebensversicherungsbank für Deutschland zu Gotha von 1828, abgedruckt in: Deutscher Verein für Versicherungs-Wissenschaft (Hg.), Sammlung von Versicherungsbedingungen Deutscher Versicherungsanstalten, Bd. II Lebensversicherung, 1909, 2f.; §§ 27f. der Statuten der LebensversicherungsGesellschaft zu Leipzig von 1830, abgedruckt in: ebd., 8f.; §16 der Statuten der 
die Rechtsfolgen bei Verstoß wurden nahezu einheitlich und identisch wie in England geregelt. So heißt es in der Verfassung der Lebensversicherungsbank für Deutschland zu Gotha von 1828 ${ }^{116}$ ):

„§60. Wenn sich nach erfolgter Aufnahme eines Versichernden finden sollte, daß er bei seiner Deklaration und in Ansehung der beigebrachten Zeugnisse nicht aufrichtig und ehrlich zu Werke gegangen wäre, so verliert er sein Recht an die Bank, ingleichen die etwa schon eingezahlten Gelder.“

Variationen gab es hinsichtlich der Frage, ob ein objektiver Verstoß gegen die Verhaltensanforderungen für den Eintritt dieser Rechtsfolge ausreichte. Der gerade zitierte $\S 60$ legt nahe, dass ein Verschulden hinzutreten musste. Der Versicherungsnehmer musste ,nicht aufrichtig und ehrlich zu Werke gegangen“ sein. Die Statuten anderer Versicherer schienen dagegen einen objektiven Verstoß ausreichen zu lassen ${ }^{117}$ ).

c) Zwischenergebnis:

Die Entwicklung der Verhaltensanforderungen, die an den Versicherungsnehmer vor Abschluss einer Lebensversicherung gestellt wurden, und die Entwicklung der Rechtsfolgen bei ihrer Verletzung verliefen in Deutschland und England parallel. Doch sind solche Parallelen nicht erst seit dem 19. Jahrhundert nachweisbar. Für diese Zeit überraschen Parallelen nicht, waren doch viele der Akteure, die im frühen 19. Jahrhundert in Deutschland die Gründung kaufmännisch betriebener Lebensversicherungen vorangetrieben hatten, mit der englischen Versicherungspraxis in Berührung gekommen ${ }^{118}$ ). Parallelen lassen sich vielmehr bereits seit dem ausgehenden 17. Jahrhundert für die Vorläufer der modernen Lebensversicherungen erkennen. Das überrascht: Zum einen zeigt dieser Befund, dass der Forschungsansatz der deutschen Literatur, der außerhalb der Seeversicherung zumeist von einer regionalen Perspektive geprägt ist ${ }^{119}$ ), wichtige Entwicklungen übersieht.

Mecklenburgschen Lebensversicherungs- und Sparbank von 1853, abgedruckt in: ebd., 12; §§ 32f. der Statuten der Lebensversicherungs- und Ersparnisbank in Stuttgart von 1854, abgedruckt in: ebd., 17. Vgl. weiters die Deklaration des Versicherungsnehmers, die Arztinstruktion und den Vordruck eines ärztlichen Zeugnisses der Gothaer Lebensversicherungsbank aus dem Jahre 1833, abgedruckt in: Heinrich Braun, Urkunden und Materialien zur Geschichte der Lebensversicherung und der Lebensversicherungstechnik, 1937, 49ff.

${ }^{116)}$ Zitiert aus Sammlung II (Fn. 115), 4.

${ }^{117}$ ) Vgl. §35 der Statuten der Lebensversicherungs-Gesellschaft zu Leipzig von 1830, abgedruckt in Sammlung II (Fn. 115), 9; Grundregeln der Deutschen Lebensversicherungs-Gesellschaft zu Lübeck, abgedruckt in: ebd., 21.

${ }^{118}$ ) Siehe oben den Text zu und die Nachweise in Fn. 64-66.

${ }^{119}$ ) Siehe oben den Text zu und die Nachweise in Fn. 54 und 77. 
Zum anderen zeigt sich, dass die in der deutschen Literatur als so wichtig erachtete Grenzziehung zwischen der genossenschaftlichen Absicherung sowie den staatlichen betriebenen oder initiierten Versicherungen einerseits und den gewerblich betriebenen Versicherungen andererseits ${ }^{120}$ ) für die Entwicklung der Verhaltensanforderungen in den Versicherungsbedingungen der Lebensversicherer und ihrer Vorläufer ohne besondere Bedeutung war.

2. Verhaltensanforderungen im Feuerversicherungswesen: Nicht nur bei Vertragsanbahnung treffen den Versicherungsnehmer Verhaltensanforderungen, sondern auch nach Eintritt des Versicherungsfalls. Im Feuerversicherungswesen muss er etwa den Versicherungsfall anzeigen, Schadensnachweise liefern und bei der Ursachenaufklärung mitwirken.

a) Entwicklung in England:

1676 wurde mit der Hamburger General-Feuer-Cassa die erste staatliche Feuerversicherung gegründet, und 1681 wollte auch die City of London eine Feuerversicherung gründen ${ }^{121}$ ). Zwei identische Vorstöße in Hamburg und London: Man möchte an keinen Zufall glauben und vermutet zahlreiche Parallelen. Bei einem Vergleich der Ordnung der Hamburger Kasse ${ }^{122}$ ) mit den Bedingungen der Londoner Versicherung ${ }^{123}$ ), finden sich aber kaum Ähnlichkeiten, welche die These einer gegenseitigen Beeinflussung stützen würden. Die City of London stellte bereits 1682 ihre Aktivitäten wieder ein und überließ das Feuerversicherungswesen privaten Akteuren.

Die verfügbaren Materialien zu den ersten unternehmerisch betriebenen englischen Feuerversicherungen deuten darauf hin, dass die Bedingungen an den Versicherungsnehmer keine Verhaltensanforderungen nach Eintritt des Versicherungsfalls stellten ${ }^{124}$ ). Doch schon zu Beginn des 18. Jahrhunderts bildeten sich solche Anforderungen heraus. Die Bedingungen der Company of London Insurers aus dem Jahr 1710, die 1708 zunächst als Exchange House Fire Office gegründet worden war, später als Sun Fire Office und dann als The Sun Insurance Office firmierte ${ }^{125}$ ), bestimmten ${ }^{126}$ ):

„Article 9. As soon as any person insured shall have his or her house or goods damaged by Fire, he or she is to give notice to the Company's Clerk at their Office [...].

$\left.{ }^{120}\right)$ Siehe vor allem oben den Text zu und den Nachweis in Fn. 48.

${ }^{121}$ ) Vgl. zum Folgenden oben den Text zu und die Nachweise in Fn. 56-59.

${ }^{122}$ ) Abgedruckt in W. Ebel, Hamburger Feuerkontrakte (Fn. 58), 83ff.

${ }^{123}$ ) Abgedruckt in Jenkins/Yoneyama (Fn. 98), I 35f.

${ }^{124}$ ) Ausgewertet wurden die Materialien in Jenkins/Yoneyama (Fn. 98) I.

${ }^{125}$ ) Zur Geschichte dieser Versicherung Peter George Muir Dickson, The Sun Insurance Office 1710-1960, 1960.

${ }^{126}$ ) Zitiert aus Jenkins/Yoneyama (Fn. 98), I 126. 
Article 11. Every Sufferer must make out his or her Loss and damage upon Oath before a Judge or Master in Chancery, in the presence of the Clerk of the Company within 10 days after the fire, and carry that Affidavit to the Minister or Churchwardens of the parish in which the Fire broke out, and some other eminent Housekeepers, in the said parish, especially such as live near the place where the fire began but have themselves sustained no damage thereby, and are best acquainted with the person, reputation and circumstances of the said Sufferer, who shall sign a certificate they do know or believe nothing to the contrary, but that the Sufferer has really and by misfortune lost by fire the sum mentioned in his or her Affidavit, upon producing which to the Company he or she shall receive his or her claim. But if there appears any fraud or perjury in such Sufferer he or she shall be excluded from any right or Interest in these proposals.“

Die Bedingungen verlangten also nicht nur eine Anzeige des Versicherungsfalls durch den Versicherungsnehmer, dieser musste zudem zur Schadenshöhe einen Eid vor einer öffentlichen Stelle abgeben und schließlich ein Leumundszeugnis durch den Pfarrer der Gemeinde oder die Gemeindevorsteher beibringen. Diese Verhaltensanforderungen hielten sich im Kern bis in das 19. Jahrhundert hinein, freilich mit leichten Variationen: Im Jahr 1716 etwa erscheint in den Bedingungen der Sun Fire Office anstelle der Frist von zehn eine solche von 15 Tagen, und das Leumundszeugnis durch den Pfarrer oder die Gemeindevorsteher konnte ausnahmsweise durch das einer Amtsperson ersetzt werden ${ }^{127}$ ); im Jahr 1726 verlangten die Bedingungen neben dem Eid und dem Leumundszeugnis die Vorlage von Büchern und Belegen, um den Schadensnachweis zu führen ${ }^{128}$ ). Bis zum Jahr 1794 fand eine Regel zu den Rechtsfolgen bei Verstoß gegen diese Verhaltensanforderungen Eingang in die Bedingungen ${ }^{129}$ ):

„Article XI. Persons Insured, sustaining any Loss or Damage by Fire, are forthwith to give Notice thereof at the Office, and as soon as possible afterwards deliver in as particular an Account of their Loss and Damage as the Nature of the Case will admit of, and make Proof of the same by their Oath or Affirmation, according to the Form practised in the said Office, and by their Books of Accompts, or other proper Vouchers, as shall be reasonably required, and procure a Certificate under the hands of the Minister and Church-Wardens, together with some other reputable Inhabitants of the Parish, not concerned in such Loss, importing, That they are well acquainted with the Character and Circumstances of the Person or Persons insured, and do know or verily believe, that he, she, or they, really and by Misfortune, without any Fraud or evil Practice, have sustained, by such Fire, the Loss and Damage, as his, her or their, Loss, to the Value therein-mentioned; but, till such Affidavit and

\footnotetext{
${ }^{127}$ ) Abgedruckt in Jenkins/Yoneyama (Fn. 98), I 127f.

${ }^{128}$ ) Abgedruckt in Jenkins/Yoneyama (Fn. 98), I 129ff.

${ }^{129}$ ) Zitiert aus Jenkins/Yoneyama (Fn. 98), I 147.
} 
Certificate of such the Insured's Loss shall be made and produced, the Loss-Money shall not be payable.“

Identische Verhaltensanforderungen waren bei allen englischen Versicherern üblich und hielten sich im 19. Jahrhundert. Nur die Anforderung, dass ein Leumundszeugnis vorgelegt werden musste, wurde im Verlauf des 19. Jahrhunderts unüblich ${ }^{130}$ ).

\section{b) Entwicklung in Deutschland:}

Unternehmerisch betriebene Feuerversicherungen gab es in Deutschland zunächst nicht ${ }^{131}$ ). Das Immobiliarfeuerversicherungswesen lag in öffentlicher Hand: Feuerversicherungen wurden vom Staat betrieben oder gingen auf seine Initiative zurück. Als Folge stellte sich das Problem, wie der Versicherer vom Versicherungsfall und von der Schadenshöhe Kenntnis erlangt und Gewissheit darüber erhält, dass der Versicherungsfall nicht in betrügerischer Absicht herbeigeführt worden ist, ganz anders dar. So bestimmte die Ordnung der Hamburger General-Feuer-Cassa von 1676 $6^{132}$ ):

„8. Solte einem sein Hauss durch Feurs-Brunst/ so GOTT gleichfalls gnädig abwenden wolle/ beschädigt werden/ so sol derselbe Schade durch die verordnete Herren und Bürgere der Feur-Ordnung/ mit zuziehung einiger Zimmer- und Maur-Leuten/ so absonderlich dazu beeidigte/ getaxiret, und alsdann der Schade aus der FeurOrdnungs-Cassa auch bezahlet werden“.

Wer Verordnete waren, legte die Ordnung nachfolgend fest ${ }^{133}$ ):

„14. Sollen allemahl die Verordneten dieser Ordnung seyn, nemlich zwee Herren des Rahts/ zwee Oberalten/ zwee Cämerei-Bürgere/ und dann noch zehn feine ehrliche Bürgere/ und zwar aus jeder Colonelschaft zwee/ nebenst einen gewissen Schreiber [...].“

Die Ordnung enthielt keine Verhaltensanforderungen, vielmehr wurden die Verordneten der Feuerkasse von Amts wegen tätig. Zur gleichen Zeit schwiegen die Bedingungen englischer Versicherer dazu, wie der Schaden festgestellt werden sollte. Es handelte sich freilich nicht um eine Innovation der Hamburger Feuerkasse von 1676. Vielmehr hatte es bereits zuvor in Hamburg sogenannte Feuerkontrakte gegeben, und die Feuerkasse ist aus diesen hervorgegangen ${ }^{134}$ ). In ihnen hatten sich Hauseigentümer zusammengeschlossen,

${ }^{130}$ ) Vgl. die Hinweise bei George Morley Dowdeswell, The Law of Life and Fire Insurance, 1846, 510ff.

131) Vgl. Koch, Geschichte (Fn. 14), 5.

${ }^{132}$ ) Zitiert aus W. Ebel, Feuerkontrakte (Fn. 58), 84.

133) Zitiert aus W. Ebel, Feuerkontrakte (Fn. 58), 84f.

${ }^{134}$ ) Zu ihnen W. Ebel, Feuerkontrakte (Fn. 58), 5ff., 31ff.; Koch, Geschichte (Fn. 14), 29-30; v. Zed twitz (Fn. 46), 126ff.; Helmer (Fn. 27), 32ff. Über die Vorbilder der Feuerkontrakte, etwa in Holland oder Schleswig-Holstein, ist viel speku- 
um Feuerschäden gemeinsam zu tragen. Und schon die Kontrakte von 1591, 1622 und 1640 enthielten Bestimmungen, wonach der Schaden von Ratsleuten und Handwerkern besichtigt und taxiert werden sollte ${ }^{135}$ ). In Deutschland wurden also zunächst keine Verhaltensanforderungen an den Versicherungsnehmer gestellt. Vielmehr wurden öffentliche Stellen von Amts wegen tätig. Nur am Rande sei bemerkt: Die für die Entwicklung des Versicherungswesens so wichtige Zäsur, dass im Jahre 1676 erstmalig eine staatlich betriebene Feuerkasse entstanden ist, ist für die Entwicklung der hier untersuchten Frage ohne Bedeutung: Die Schadensfeststellung erfolgte vorher und nachher auf identische Weise.

An dem gerade aufgezeigten Zustand änderte sich auch nachfolgend zunächst nichts. So bestimmte etwa das Patent der Brand-Assecurations-Societät im Fürstentum Weimar aus dem Jahre $1768^{136}$ ):

„15) wenn in dem hiesigen Fürstenthum [...] sich nach Publication gegenwärtiger Unserer Verordnung ein Unglück ereignen und dieser oder jener Ort mit Feuer heimgesucht werden würde, welches doch göttliche Güte von Unsern getreuen Unterthanen möglichst abwenden wolle! alsdann davon durch die Obrigkeit des Orts, mit specifiquer Bemerkung der abgebrannten und beschädigten Gebäude nach denen Nummern und Ziffern, womit selbige in dem Cataster eingezeichnet sind, ohnverzüglich die berichtliche Anzeige an die angeordnete Deputation geschehen solle.“

Die Anzeige des Brands erfolgte durch die Obrigkeit des Orts. Diese fertigte sodann einen Bericht über die Brandursache und den Schaden an. Das Patent fährt fort ${ }^{137}$ ):

liert worden, ohne dass Vorläufer nachgewiesen werden konnten. Vgl. die Darstellungen bei W. Ebel, Feuerkontrakte (Fn. 58), 31ff.; Helmer (Fn. 27), 6ff.; S chaefer (Fn. 25), 163; Spi es (Fn. 69), 251; Franz Büchner, Die Entstehung der Hamburger Feuerkasse und ihre Entwicklung bis zur Mitte des 19. Jahrhunderts, in: 300 Jahre Hamburger Feuerkasse, 1976, 1-37, 5; Schmitt-Lermann (Fn. 70), 6; Franz Maria Heibl, Geschichte der Brandversicherung in Bayern, 1938, 14; Hamburger Feuerkasse (Fn. 29), 14; Morstein Marx (Fn. 57), Sp. 648ff. Zu den Brandgilden in Schleswig-Holstein siehe etwa Ludwig Maass, Die Brandgilden insbesondere in Schleswig Holstein, 1909. Kritisch die Diskussionen zusammenfassend Zwierlein (Fn. 50), 35, $226 f$.

$\left.{ }^{135}\right)$ Sie sind abgedruckt bei W. Ebel, Feuerkontrakte (Fn. 58), 66ff. Weitere Beispiele sind abgedruckt bei Schaefer (Fn. 25), 202ff.

${ }^{136)}$ Patent, die in dem Fürstenthum Weimar und in der Jenaischen Landes-Portion zu errichtenden Brand-Assecurations-Societät betreffend vom 23. August 1768, abgedruckt in: Johannes Schmidt (Hg.), Aeltere und neuere Gesetze, Ordnungen und Circular-Befehle für das Fürstenthum Weimar und für die Jenaischen Landes-Portion bis zum Ende des Jahres 1799, Bd. I, 1800, 498f.

${ }^{137}$ ) Patent (Fn. 136), 501. 
„18) Die Untersuchung der Veranlassung zu einem entstandenen Brand-Schaden verbleibt der Obrigkeit jeden Orts, so ferne selbige die Befugnis, dergleichen Untersuchungen zu verführen, und nach Befinden der Umstände die Bestrafung der schuldig befundenen zu bestimmen, hergebracht hat. Es hat jedoch selbige von dem, was sich bey der Untersuchung ergeben, auch bey der Deputation zu dem Ende die Anzeige zu thun, damit selbige dadurch in den Stand gesetzet werde, zu ermessen, ob und in wie weit bey dem vorgefallenen Brande eine Bosheit oder aber eine derselben gleich zu rechnende Fahrlässigkeit vorgekommen, mithin, ob dem Beschädigten eine Entschädigung gebühre, und prästiret werden müsse oder nicht. Denn da ist Unsere Intention, daß derjenige, der vorsetzlich eine Feuersbrunst veranlasset, außer der ihm als einem Mordbrenner zu dictirenden Strafe, auch noch von allem Genuß einiger Entschädigung ausgeschlossen seyn $[\ldots]$ solle.“

Ganz ähnliche Bedingungen finden sich in anderen Ordnungen der öffentlich-rechtlichen Feuerkassen des 18. Jahrhunderts. Überall erfolgte die Anzeige des Versicherungsfalls, die Ermittlungen der Schadensursache, die Schätzung der Schadenshöhe und die Anfertigung der entsprechenden Protokolle durch öffentliche Stellen auf Grundlage der entsprechenden Ordnungen $^{138}$ ). Die Versicherungsbedingungen in England und Deutschland haben nach alledem mit Blick auf die an den Versicherungsnehmer gestellten Verhaltensanforderungen bis zum Ende des 18. Jahrhunderts unterschiedliche Wege beschritten.

Aber wie war es im 19. Jahrhundert? Seit dem 19. Jahrhundert drangen englische Versicherer auf den deutschen Markt ${ }^{139}$ ), so die Sun Fire Insurance seit dem Jahr 1836 ${ }^{140}$ ). Die 1782 gegründet Phoenix Assurance Company nahm ihr Geschäft in Hamburg 1786 auf und soll von hier aus den deutschen Markt beherrscht haben ${ }^{141}$ ). Zeitgleich gründeten sich in Deutschland die ers-

138) Vgl. etwa Art. IX der Kurfürstlich Mainzischen Assecuranz-Ordnung von 1780, abgedruckt in Hans Meister, Geschichte der Immobiliar-Brand-Versicherung in Unterfranken, 1913, 74f.; § 3 der Fürstbischöflichen Würzburger Feuerassekuranz-Ordnung von 1768, abgedruckt in: ebd., 58f.; Art. XI der Verordnung der Braunschweigschen Brandassecurations-Societät von 1750, abgedruckt in: ebd., 91f.; Vorderösterreichische Feuer-Societäts-Ordnung von 1764, abgedruckt in: Max Leimdörfer, Entwicklung und Organisation der Brandversicherung in Österreich 1700-1848, 1905, 240; § IV der Kur-Badischen neuen Brandversicherungs-Ordnung von 1804, abgedruckt in: Deutscher Verein für Versicherungs-Wissenschaft (Hg.), Sammlung von Versicherungsbedingungen Deutscher Versicherungsanstalten, Bd. I, 1908, 45. Siehe weiterhin die Darstellung bei Heibl (Fn. 134), 36, 45, 52, 70.

$\left.{ }^{139}\right)$ Vgl. oben den Text zu und nach Fn. 60.

${ }^{140}$ ) Dickson (Fn. 125), 163ff.; Raynes (Fn. 17), 258; Cockerell/Green (Fn. 18), 25; Zwierlein (Fn. 50), 324ff.

${ }^{141}$ ) Koch, FS Lorenz (Fn. 25), 423; ders., Geschichte (Fn. 14), 47ff.; Müs s ener 
ten unternehmerisch betriebenen Feuerversicherungen ${ }^{142}$ ). Betrachten wir vor diesem Hintergrund die Versicherungsbedingungen der deutschen Feuerversicherer und beginnen beispielhaft mit der Verfassung der Berlinischen Feuerversicherungs-Anstalt von $1812^{143}$ ):

„Art. 42. Bei eingetretener Feuersbrunst ist der Versicherte [...] verpflichtet, von dem eingetretenen Feuerschaden sogleich eine vorläufige Anzeige an die Anstalt oder deren Agenten zu machen. Wer diese Anzeige nicht spätestens innerhalb vier Wochen, vom Tage des Brandes an gerechnet, macht, verliert allen Anspruch auf Schadensersatz. [...]

Art. 44. Zum Beweis des Schadens wird erfordert: 1. Eine gerichtliche Verhandlung über die gleich nach dem Brande zu veranstaltende Untersuchung der Ursachen des Feuers. 2. Im Fall der versicherte Gegenstand ganz verbrannt ist, eine von der Gerichtsbehörde über den Beweis aufgenommene Verhandlung. 3. Bei Partialschäden an Gebäuden und Schiffen, eine unter Leitung der Gerichtsbehörde durch zwei Sachverständige aufgenommene und an Eidesstatt unterzeichnete Spezifikation und Taxation des Schadens und desjenigen, was beschädigt oder unbeschädigt nachgeblieben ist. 4. Bei Partialschäden an Waren, Fabrikaten und Fabrikgerätschaften, ein befriedigender Beweis über die Wirklichkeit und Größe des Schadens, welcher etwas geführt werden könnte: durch Handlungsbücher, letzte Bilanz oder Inventarium, durch Original-Rechnungen, durch beglaubigte Erklärungen des Eigentümers, seiner Leute und solcher Personen, welche das Eigentum kannten und den Wert der verbrannten und beschädigten Güter gehörig zu beurteilen imstande sind. Auf Verlangen müssen alle diese Beweismittel eidlich bestärkt werden. 5. Bei Partialschäden an Möbeln und Hausgeräten, eine Spezifikation der verbrannten und beschädigten Sachen und deren Wertes, welche durch eine Erklärung an Eides Statt bekräftigt und mit Zeugnissen von glaubwürdigen Personen begleitet sein muß.“

In den deutschen Bedingungen des 18. Jahrhunderts ließen sich keine Anforderung an den Versicherungsnehmer finden, wonach er den Versicherungsfall anzeigen musste. Art. 42 der Verfassung der Berlinischen Anstalt kannte eine solche Anforderung. Der Versicherungsfall musste in den englischen Bedingungen „as soon as possible“, hier „sogleich“ angezeigt werden, wobei die Berlinische Anstalt noch eine Obergrenze von vier Wochen einführte. Das ist zunächst wenig überraschend: Der Gründer der Feuerversicherungs-Anstalt, Georg Friedrich Averdieck (1774-1839), war zuvor Vertreter der Phoenix gewesen; auch der bereits erwähnte Benecke ${ }^{144}$ )

(Fn. 57), 33. Zum Auslandsgeschäft der Phoenix vgl. Trebilcock (Fn. 30), 162ff., 187ff.; Raynes (Fn. 17), 257f.; Cockerell/Green (Fn. 18), $24 f$.

${ }^{142}$ ) Vgl. etwa die Darstellung von Müss ener (Fn. 57), 33ff.

${ }^{143}$ ) Zitiert aus Sammlung I (Fn. 138), 23f. Zu dieser Anstalt vgl. nur Denkschrift zum hundertjährigen Bestehen der Berlinischen Feuer-Versicherungs-Anstalt, 1912.

${ }^{144}$ ) Siehe oben den Text zu Fn. 64. 
hatte bei der Gründung mitgewirkt; und als Folge geht die deutsche Literatur davon aus, dass sich die Bedingungen der Berlinischen Anstalt an die der Phoenix anlehnten ${ }^{145}$ ). Aber wie die älteren deutschen Vorbilder erforderte die Verfassung weiterhin eine gerichtliche Untersuchung und Schadensermittlung durch Sachverständige. Eine bloße Imitation oder Übersetzung der Bedingungen der Phoenix liegt also nicht vor. Wie in den englischen Bedingungen war der Versicherungsnehmer freilich angehalten, Schadensaufstellungen und seine Geschäftsbücher vorzulegen und alle Angabe eidlich zu bestärken. Die Rechtsfolgen regelte die Verfassung der Berlinischen Anstalt in Art. 50 nur ganz allgemein ${ }^{146}$ ):

„Art. 50. Der Bevollmächtigte oder die Agenten haben die Befugnis, Nachfragen und Untersuchungen anzustellen, welche sie während der Versicherung oder bei einem Schaden für erforderlich halten [...]. Wer diesen Nachfragen und Untersuchungen sich nicht gutwillig unterwirft, oder sich weigert, gehörige Auskunft zu erteilen, verliert allen Anspruch auf Schadensersatz und die Prämie ist verfallen.“

Dass es anders ging, zeigen die Statuten der württembergischen PrivatFeuerversicherungs-Gesellschaft von 1828, die sich noch ganz an die älteren deutschen Vorbildern halten ${ }^{147}$ ): Der Versicherungsnehmer war zwar gehalten, den Versicherungsfall innerhalb von 24 Stunden anzuzeigen und vorläufig den ungefähren Schaden zu schätzen. Im Übrigen lag die Schadensermittlung aber in der Hand des Agenten, der auch offizielle Stellen, wie Polizeibehörden, um die Übermittlung von etwaigen Untersuchungsprotokollen zu ersuchen hatte. Die Rechtsfolge eines Verstoßes gegen die Anforderung, den Brand innerhalb von 24 Stunden anzuzeigen, regelten diese Statuten freilich eindeutig: Der Versicherungsnehmer büßte seinen Anspruch auf Entschädigung ein.

Die Allgemeinen Versicherungs-Bedingungen der Aachener und Münchener Feuerversicherungs-Gesellschaft von 1829 verlagerten dagegen die Schadensermittlung ganz in die Sphäre des Versicherungsnehmers ${ }^{148}$ ): Er musste den Versicherungsfall innerhalb von 24 Stunden anzeigen, sich innerhalb von 24 Stunden bei einer Ortspolizeibehörde einfinden, um sich über Schadensursache und -höhe vernehmen zu lassen, und sodann das darüber aufgenommene Protokoll innerhalb von zehn Tagen an den Versicherer einsenden. Erfüllte er diese Verhaltensanforderungen nicht fristgerecht, verlor er

${ }^{145}$ ) Lammel (Fn. 22), 103; Koch, Geschichte (Fn. 14), 49, 63; ders., FS Lorenz (Fn. 25), 423f.

$\left.{ }^{146}\right)$ Zitiert aus Sammlung I (Fn. 138), 25.

${ }^{147}$ ) Abgedruckt in: Sammlung I (Fn. 138), 14-22.

${ }^{148}$ ) Abgedruckt in: Sammlung I (Fn. 138), 26-30. 
seine Ansprüche. Diese Bedingungen konsolidierten sich und wurden üblich. So bestimmten die Allgemeinen Versicherungs-Bedingungen der Magdeburger Feuerversicherungs-Gesellschaft von $1845^{149}$ ):

„§9. Bei eingetretener Feuersbrunst ist der Versicherte verpflichtet: [...]

b) bei Verlust seiner Ansprüche auf Schadensersatz:

1. binnen 24 Stunden von dem eingetretenen Feuerschaden vorläufige Anzeige an den [...] Agenten zu machen;

2. binnen drei Tagen über die mutmaßlich Entstehung des Feuers und alle dasselbe betreffenden näheren Umstände, bei beweglichen Gegenständen auch zugleich über die Art und ungefähre Höhe des Schadens vor seiner Ortspolizeibehörde sich vernehmen zu lassen;

3. binnen vierzehn Tagen beglaubigte Abschrift des Protokolls dem Agenten auf zuverlässigen Wege einzusenden und zugleich demselben bei Versicherungen von beweglichen Gegenständen eine spezielle Schadensrechnung einzureichen, begleitet von einer möglichst detaillierten Nachweisung aller Gegenstände, welche sich zur Zeit des Brandes in den in der Police bezeichneten Lokalen befanden, unter Angabe, welche von diesen Gegenständen völlig verbrannt, welche beschädigt wurden und welche unbeschädigt blieben. [...]

$\S 18$. Alle nicht innerhalb Jahresfrist nach dem Brande entweder festgestellten oder vor Schiedsrichter gebrachten Ansprüche auf Entschädigung sind erloschen.“

Freilich lassen sich auch Kontinuitäten zu den älteren deutschen Bedingungen erkennen, war doch weiterhin die Einschaltung öffentlicher Stellen notwendig. Aber diese wurden eben nicht mehr von Amts wegen tätig. Im Unterschied zu den englischen Bedingungen waren keine Leumundszeugnisse nötig. Auch das lag wohl daran, dass in Deutschland öffentliche Stellen eingebunden waren. Wie in englischen Bedingungen war der Versicherungsnehmer nach vielen deutschen Bedingungen gehalten, Geschäftsbücher vorzulegen, und alle Angaben eidlich zu bestärken. Übereinstimmungen zur englischen Versicherungspraxis finden sich schließlich auch in der Rechtsfolge: Hat der Versicherungsnehmer gegen die Verhaltensanforderungen verstoßen, so verlor er seine Ansprüche.

c) Zwischenergebnis:

Im Feuerversicherungswesen verlief die Entwicklung der Verhaltensanforderungen in England und Deutschland zunächst nicht parallel. Vielmehr hatten die Entwicklungen am Ende des 17. Jahrhunderts unterschiedliche Ausgangspunkte, und folgten im 18. Jahrhundert unterschiedlichen Linien. Im 19. Jahrhundert kam es zu einer Angleichung der deutschen Verhaltensanforderungen an die der englischen Praxis. Aber die Bedingungen der englischen Versicherer wurden in Deutschland nicht einfach imitiert. Vielmehr

${ }^{149}$ ) Zitiert aus Sammlung I (Fn. 138), 35f. 
zeigen sich in den Bedingungen des 19. Jahrhunderts in Deutschland noch Elemente aus den älteren deutschen Bedingungen. Nur am Rande sei bemerkt: Cornel Zwierlein arbeitete für die Entwicklung des Versicherungswesens das ausgehende 17. und frühe 18. Jahrhundert als wichtige Zäsur heraus. Er spricht von einer Epochenschwelle der Sicherheitsregime um 1680/1700 und macht diese an der Entwicklung der Feuerversicherung in London und Hamburg fest ${ }^{150}$ ). So wichtig diese Zäsur für die von Zwierlein untersuchte Frage auch gewesen sein mag, für die Entwicklung der Bedingungen der Feuerkontrakte und Feuerkassen in Deutschland war sie mit Blick auf die hier betrachtete Frage nur von untergeordneter Bedeutung, knüpften doch die Bedingungen der Hamburger General-Feuer-Cassa von 1676 unmittelbar an die Bedingungen der Feuerkontrakte an, wie sie sich seit dem ausgehenden 16. Jahrhundert etabliert hatten.

3. Rechtsprechung zu Verhaltensanforderungen im Feuerversicherungswesen:

Aber sind die in den vorangegangen Abschnitten aufgezeigten Ähnlichkeiten nicht zu gering, als dass sie einen Einfluss der englischen auf die deutsche Praxis oder vielleicht eine gegenseitige Beeinflussung nahe legen? Könnte es sich nicht um selbständige Parallelentwicklungen handeln? Vor dem Hintergrund dieser Zweifel soll die Rechtsprechung zu den Verhaltensanforderungen nach Eintritt des Versicherungsfalls im Feuerversicherungswesen beleuchtet werden ${ }^{151}$ ).

a) Entwicklung in England:

Für England ist die Rechtslage seit dem ausgehenden 18. Jahrhundert eindeutig. Die Verhaltensanforderungen wurden als Bedingungen, und zwar als Bedingungen im Sinne von $\S 158$ BGB, eingeordnet. Beispielhaft wird dies in zwei Entscheidungen deutlich. Die erste ist Worsley v. Wo od aus dem Jahre 1796. Streitentscheidend war die Bedingung, dass der Versicherungsnehmer Leumundszeugnisse beizubringen hatte ${ }^{152}$ ). Doch weigerten sich Pfarrer und Gemeindevorsteher solche auszustellen. Der Versicherungsnehmer meinte, dass von ihm nur verlangt werden könne, dass er sich redlich um ihre Ausstellung bemühe. Ob sie ausgestellt werden, liege dagegen außerhalb seiner Macht. Ashhurst J. stellte klarris3):

${ }^{150}$ ) Zwierlein (Fn. 50), 198ff.

$\left.{ }^{151}\right)$ Vgl. zum Folgenden schon Phillip Hellwege, Obliegenheiten im Versicherungsvertragsrecht aus historisch-vergleichender Perspektive, RabelsZ 76 (2012), 864-892.

152) Für eine vergleichbare Bedingung siehe oben die Zitate zu Fnn. 126 und 129.

153) (1796) 6 Term Reports 710, 720 per Ashhurst J. 
„The question is whether the certificate required be or be not a condition precedent; if it be, the plaintiffs below cannot maintain their action. It is perfectly immaterial whether they have entered into an improvident contract; if they choose to take the burden upon themselves, they cannot call on the insurance office until they have complied with the condition. I think it is a condition precedent [...]. Nor is there any thing unreasonable in these terms.“

Daraus folge, dass die Bedingung exakt zu erfüllen sei. Nur dann komme der bedingte Anspruch auf die Versicherungsleistung überhaupt erst zur Entstehung. Die zweite Entscheidung ist Mason v. Harvey aus dem Jahre 1853. Hier ging es um die Bedingung, dass der Versicherungsnehmer fristgerecht eine Schadensaufstellung einzureichen hatte ${ }^{154}$ ). Der Versicherungsnehmer argumentierte, dass es sich nicht um eine Bedingung handeln könne, weil dann jede noch so unbedeutende Ungenauigkeit dazu führe, dass die Bedingung nicht erfüllt sei und der Anspruch auf die Versicherungsleistung nicht entstehe. Pollock C.B. führte aus ${ }^{155}$ ):

„By the contract of the parties, the delivery of the particulars of loss is made a condition precedent to the right of the asured to recover."

Die Erfüllung der Verhaltensanforderungen war Bedingung dafür, dass ein Anspruch gegen die Versicherung entstand. Als Folge war es unbeachtlich, ob der Versicherungsnehmer die Bedingung nicht erfüllen konnte, ob ihre Erfüllung nutzlos war oder ob er die Bedingung schuldlos nicht erfüllt hat ${ }^{156}$ ). Diese Rechtslage hat sich bis heute im Grundsatz gehalten ${ }^{157}$ ).

b) Entwicklung in Deutschland:

Auch die deutschen Gerichte gingen bis etwa 1865 davon aus, dass die eben vorgestellten Verhaltensanforderungen Bedingungen seien, wie in zwei Urteilen beispielhaft deutlich wird ${ }^{158}$ ). Für das Urteil des Rheinischen Appellationsgerichtshofs aus dem Jahre 1847 war die Klausel streitentscheidend, dass der Anspruch auf die Versicherungsleistung erlischt, wenn er nicht innerhalb einer bestimmten Frist, hier waren es sechs Monate, gerichtlich geltend gemacht werde ${ }^{159}$ ). Der Kläger berief sich darauf, dass ihm eine fristgerechte Klageerhebung aus Gründen, die nicht in seiner Macht gelegen hätten, unmöglich gewesen sei. Das Gericht führte aus ${ }^{160}$ ):

${ }^{154}$ ) Für eine vergleichbare Bedingung siehe oben das Zitat zu Fn. 129.

155) (1853) 8 Exchequer Reports 819, 821 per Pollock C.B.

156) Vgl. ausführlich die Darstellung bei Hellwege (Fn. 151), 873ff.

${ }^{157}$ ) Vgl. beispielhaft die Darstellungen bei Nicholas Legh-Jones/Johns Birds/David Owen, MacGillivray on Insurance Law, 11. Aufl. 2008, §§19-034ff.

${ }^{158}$ ) Zur Rechtslage in Deutschland bis etwa 1865 Hellwe ge (Fn. 151), 877ff.

${ }^{159}$ ) Für eine vergleichbare Bedingung siehe oben das Zitat zu Fn. 149 unter § 18.

${ }^{160}$ ) Rheinischer Appellationsgerichtshof zu Köln (28. 6. 1847), Archiv für das Ci- 
„Daß es zu der Begründung der Anwendbarkeit dieser vertragsmäßigen Uebereinkunft der Parteien, einer vorherigen Versetzung des Appellaten in Verzug keineswegs bedurfte, da es sich hier [...] von der Erfüllung einer verabredeten Bedingung handelt, ohne deren Vorhandensein der Appellat einen Anspruch auf Entschädigung überhaupt nicht geltend machen konnte [...].“

Der Kläger im Urteil des Stadt- und Kreisgerichts Danzig aus dem Jahre 1863 hatte es unterlassen, eine beglaubigte Abschrift des Protokolls über seine Vernehmung durch die Ortspolizeibehörde innerhalb von 14 Tagen an die Versicherung zu schicken ${ }^{161}$ ). Er meinte, dies sei entbehrlich gewesen, weil ein Agent der Versicherung bei der Vernehmung zugegen gewesen war. Das Gericht führte aus $\left.{ }^{162}\right)$ :

„Die Einreichung der in Rede stehenden beglaubigten Abschriften hat [...] die Bedeutung einer Bedingung, und zwar, da es sich wesentlich um eine Handlung des bedingt Berechtigten handelt, einer (positiven) Potestativ-Bedingung [...].“

Die deutschen Gerichte zogen aus der Einordnung der Verhaltensanforderungen als Bedingungen die gleichen Schlüsse wie die englischen Gerichte: Es sei unerheblich, wenn der Versicherungsnehmer die Verhaltensanforderung schuldlos nicht erfüllte $\left.{ }^{163}\right)$. Zwischen 1865 und 1870 kam es in Deutschland sodann zu einer dramatischen Rechtsprechungsänderung ${ }^{164}$ ). Ein Urteil des Ostpreußischen Tribunals zu Königsberg markiert das Ende dieser Entwicklung. Nach § 12 der entscheidungsrelevanten Bedingungen

„ist (wie dies in Policen der meisten Gesellschaften zu sein pflegt) auf so viele Versäumnisse des Versicherten die Strafe des Verlustes seiner Ansprüche gesetzt, daß nothwendigerweise angenommen werden muß, daß alle diese Verletzungen eine Schuld des Versicherten voraussetzen. Es entspricht diese Annahme auch den gesetzlichen Bestimmungen über lex commissoria, Conventionalstrafe und Verzug [...]. Ueberdies würde die Verklagte mit der entgegengesetzten Auslegung gegen $\S 2024$ II. 8 A.L.R. handeln; es würde die Treue und Redlichkeit, zu welcher auch der Versicherer verpflichtet ist, verletzen, wenn sie die Police so verstehen wollte, daß es schließlich fast ganz ihrem Belieben überlassen bliebe, ob sie die Entschädigung zahlen will. Denn auch der sorgfältigste Versicherer wird kaum vermeiden können, in einen dieser Präjudizfälle des $§ 12$ zu verfallen“165).

vil- und Criminal-Recht der Königlich-Preußischen Rheinprovinzen 42/1 (1847), 92, 95 [Betonung hinzugefügt P.H.].

${ }^{161}$ ) Für eine vergleichbare Bedingung siehe oben das Zitat zu Fn. 149 unter §9b Nr. 3.

$\left.{ }^{162}\right)$ Stadt- und Kreisgericht Danzig (3.12. 1863), Central-Organ für das deutsche Handels- und Wechselrecht 3 (1864), 65 [Betonung hinzugefügt P.H.].

163) Vgl. hierzu bereits ausführlich Hellwege (Fn. 151), 877ff.

${ }^{164}$ ) Vgl. hierzu bereits ausführlich Hellwege (Fn. 151), 882ff.

${ }^{165}$ ) Ostpreußische Tribunals zu Königsberg, Gruchots Beiträge zur Erläuterung des Deutschen Rechts 15 (1871), 556. Zur Bedeutung dieser Rechtsprechung für die Ent- 
Ein Verstoß gegen eine Verhaltensanforderung war nur noch bei einem Verschulden des Versicherungsnehmers beachtlich. Die Grundlagen für die sogenannten Obliegenheiten des modernen Rechts waren damit gelegt.

c) Zwischenergebnis:

Wer die Einordnung von Verhaltensanforderungen an den Versicherungsnehmer im modernen englischen und deutschen Recht vergleichend betrachtet, wird grundlegende Unterschiede feststellen. Während englische Gerichte solche Anforderungen bis heute als Bedingungen verstehen mit der Folge, dass dadurch erzeugte Unbilligkeiten auf ganz unterschiedliche Arten ausgeglichen werden müssen ${ }^{166}$ ), hat das deutsche Recht die Figur der Obliegenheit entwickelt. Ein Blick in die Geschichte offenbart indes, dass beide Rechte einen gemeinsamen Ausgangspunkt hatten: Auch deutsche Gerichte gingen bis etwa 1865 davon aus, dass es sich bei solchen Verhaltensanforderungen um Bedingungen handelt.

Das Beispiel zeigt zugleich die Bedeutung des allgemeinen Vertragsrechts für eine historische Vergleichung des Versicherungsrechts. In England und Deutschland stellte sich gleichermaßen die Frage, wie die in den Versicherungsbedingungen normierten Verhaltensanforderungen zu qualifizieren sind. In beiden Rechten verfiel man auf eine Figur, die aus dem allgemeinen Vertragsrecht bekannt ist, nämlich die der Bedingung. Das Bedingungsrecht hat nun seinerseits in England und Deutschland eine gemeinsame Wurzel. Die Vermutung liegt nahe, dass die englische und deutsche Rechtsprechung die Verhaltensanforderungen auch deshalb zunächst als Bedingungen einordnete, weil die englischen und deutschen Versicherer, getragen von einer gemeinsame Praxis in ihren Bedingungen, vor den nationalen Gerichten auf ähnlichen Bahnen argumentierten.

\section{Zusammenfassung}

Ausgangspunkte des Beitrags waren die Beobachtung, dass historisch-vergleichende Forschung im Versicherungsrecht weitgehend fehlt, und die daraus hergeleitete Frage, ob sie hier überhaupt möglich und zielführend ist. Sie setzt gemeinsame Wurzeln der europäischen Versicherungsrechte oder zumindest Verknüpfungen zwischen den nationalen Entwicklungen voraus. Die nationalen Forschungsstände zur Versicherungsgeschichte erwecken

wicklung des AGB-Rechts vgl. bereits allgemein Phillip Hellwege, Allgemeine Geschäftsbedingungen, einseitig gestellte Vertragsbedingungen und die allgemeine Rechtsgeschäftslehre, 2010, 172-184.

166) Hierzu vor allem Gies ela Rühl, Die englischen warranties, ZEuP 14 (2006), 607-629, 607ff.; dies. (Fn. 93), passim. 
zwar den Eindruck, als hätten sich die nationalen Versicherungsrechte weitgehend unterschiedlich entwickelt, zugleich deuten sie aber auch Verknüpfungen zwischen den nationalen Entwicklungen an.

Auf dieser Grundlage wurden sodann drei Beispiele beleuchtet, zwei aus der Versicherungspraxis in Deutschland und England und eins aus der Rechtsprechung beider Rechte. Sie zeigten, dass die Entwicklungen in der Tat Verknüpfungen aufweisen. Zwar mag man zweifelnd fragen, ob es sich nicht eher um selbständige Parallelentwicklungen gehandelt habe, die daraus resultieren, dass sich im Versicherungsrecht überall ähnliche Regelungsprobleme stellen. Doch liegt auf Grundlage der Verflechtungen der nationalen Entwicklungen, etwa der Praxis ${ }^{167}$ ), die Annahme von wirklichen Verknüpfungen näher. Gewissheit wird freilich erst die weitere Forschung bringen. Die Voraussetzungen einer historisch-vergleichenden Bearbeitung des Versicherungsrechts sind aber gegeben.

Doch welchen Beitrag kann eine solche Forschung zur Schaffung einer europäischen Versicherungsrechtswissenschaft leisten? Ihr Potenzial zeigt das Beispiel aus der Rechtsprechung: Das deutsche und englische Recht haben Verhaltensanforderungen an den Versicherungsnehmer zunächst gleichermaßen als Bedingungen begriffen und haben damit einen gemeinsamen Ausgangspunkt. Nun wird auf deutscher Seite niemand vorschlagen wollen, dass das deutsche Recht zu dieser gemeinsamen Wurzel zurückkehren soll. Auch zwingende Schlüsse, wie ein europäisches Versicherungsrecht Verhaltensanforderungen einordnen soll, ergeben sich aus dieser Erkenntnis nicht. Aber sie kann einem Dialog, hier zwischen englischen und deutschen Juristen, welche die unterschiedliche Einordnung der Verhaltensanforderungen in den geltenden Rechten als gravierend empfinden, neue Impulse geben und so den Weg hin zu einer europäischen Lösung ebnen. So können deutsche Juristen etwa darauf verweisen, dass die deutsche Rechtsprechung das Verschuldenserfordernis unter anderem daraus hergeleitet hat, dass Versicherungsverträge Treu und Glauben unterstehen ${ }^{168}$ ). Aber auch das englische Recht versteht Versicherungsverträge als Verträge of utmost good faith. Zwar sollen sich daraus für beide Parteien Treuepflichten ergeben ${ }^{169}$ ), doch hat die englische Rechtsprechung bisher einseitig diejenigen des Versicherungsnehmers betont ${ }^{170}$ ). Ob sich englische Juristen von dieser Argumentation überzeugen lassen, steht

${ }^{167)}$ Siehe oben den Text zu Fnn. 60-66.

${ }^{168}$ ) Siehe beispielhaft oben das Zitat zu Fn. 165.

${ }^{169}$ ) Vgl. Drake Insurance plc. v. Provident Insurance plc. [2003] England and Wales Court of Appeal (Civil Division) Decisions 1834.

${ }^{170}$ ) Vgl. John Birds, Modern Insurance Law, 8. Aufl. 2010, § 7.0. 
freilich auf einem anderen Blatt. Neue Impulse für einen Dialog ergeben sich aber allemal.

Schließlich stellt sich eine Folgefrage, die nur noch gestreift werden soll, nämlich die nach den Perspektiven einer historisch-vergleichenden Bearbeitung des Versicherungsrechts. Historisch-vergleichende Forschung ist quellenund zeitintensiv. Wird sie nicht von der schnell voranschreitenden Rechtsvereinheitlichung überholt werden? In vielen Branchen stärkt eine europäische Rechtsharmonisierung den Binnenmarkt. Aber sie ist nicht Grundvoraussetzung für dessen Funktionieren. Anders im Versicherungswesen. Hier ist ein europäischer Binnenmarkt nur auf Grundlage eines europäischen Versicherungsvertragsrechts möglich ${ }^{171}$ ). Denn die Versicherung ist ein „Rechtsprodukt“, „ein abstraktes Gut; sie wird ausschließlich durch Rechtstexte vergegenständlicht“"172), durch allgemeine Versicherungsbedingungen geprägt. Doch ihre allgemeinen Versicherungsbedingungen können die Versicherer nicht in ganz Europa verwenden. Dies liegt vor allem an zwingenden Bestimmungen der nationalen Versicherungsrechte. Es ist daher richtig, dass sich die Verfasser der PEICL zunächst auf das zwingende Recht beschränkt haben ${ }^{173}$ ): Die PEICL sollen als optionales Instrument den Versicherern so die Möglichkeit geben, ihre allgemeinen Versicherungsbedingungen in ganz Europa einzusetzen.

Ist also eine Vereinheitlichung der zwingenden Bestimmungen der europäischen Versicherungsrechte Grundvoraussetzung für einen funktionierenden Binnenmarkt im Bereich des Versicherungswesens, so wird sie nicht mehr lange auf sich warten lassen. Die Einführung eines optionalen Instruments scheint im Versicherungsrecht greifbar zu sein. Wird damit aber aus einem Forschungsansatz, der nach seinem Selbstverständnis eine europäische Privatrechtswissenschaft mit erschaffen soll, aus der dann erst ein europäisches Privatrecht erwachsen kann, sofort ein reines Grundlagenfach? Kann sich die europäische Versicherungsrechtswissenschaft mit anderen Worten nach Einführung eines optionalen Instruments auf dessen dogmatische Durchdringung beschränken ${ }^{174}$ )? Ich denke nicht:

Zum einen setzt ein optionales Instrument die nationalen Versicherungsrechte nicht außer Kraft. Es tritt nur neben diese. Zum anderen soll es zu-

171) Vgl. Basedow, FS Lorenz (Fn. 9), 93ff.

$\left.{ }^{172}\right)$ Meinrad Dreher, Die Versicherung als Rechtsprodukt, 1991, 3.

$\left.{ }^{173}\right)$ Hierzu Reichert-Facilides, FS Drobnig (Fn. 10), 134; Anton K. Schnyder/Ahmet Kut, Versicherungsvertrag, in: Handwörterbuch (Fn. 1), 1661-1664, 1662.

${ }^{174}$ ) Siehe hierzu schon oben den Text zu Fn. 13. 
nächst nur zwingendes Recht enthalten. Im Übrigen bleibt es bei den nationalen Versicherungsrechten. Vor allem aber muss Wissenschaft Rechtsvereinheitlichung vordenken. Schreitet der Gesetzgeber indes in einem Tempo voran, dass sich zuvor keine einheitliche Rechtswissenschaft bilden konnte, die den Boden für eine Rechtsvereinheitlichung ebnet, so gefährdet dies die einheitliche Anwendung und die Akzeptanz des vereinheitlichten Rechts. Akzeptanz wird für das vereinheitlichte Recht nur geschaffen, wenn die Unterschiede zwischen den nationalen Rechten (historisch-)vergleichend erklärt werden und so die Wahl des optionalen Instruments für eine bestimmte Lösung vermittelt wird. Eine einheitliche Anwendung des vereinheitlichten Rechts ist nur möglich, wenn sich die Rechtsanwender ihrer national geprägten und historisch bedingten Vorverständnisse bewusst werden. Mit anderen Worten stellt sich die Aufgabe der Schaffung einer europäischen Versicherungsrechtswissenschaft auch nach Einführung eines optionalen Instruments, und um diese Aufgabe zu bewältigen muss (historisch-)vergleichend gearbeitet werden. Auch nach Einführung eines optionalen Instruments bleibt die europäische Versicherungsrechtswissenschaft mithin eine im Kern komparative Wissenschaft, die um eine historische Perspektive ergänzt werden kann und sollte. 